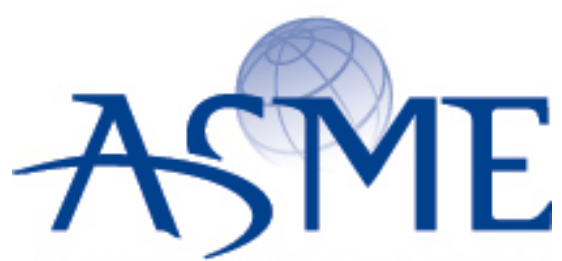

SETTING THE STANDARD

\section{American Society of Mechanical Engineers}

ASME Accepted Manuscript Repository

Institutional Repository Cover Sheet

ASME Paper Title: Dynamical Modelling and Control of Modular Snake Robots with Series Elastic

Actuators for Pedal Wave Locomotion on Uneven Terrain

Mohammadali Javaheri Koopaee, Christopher Pretty, Koen Classens,

Authors: XiaoQi Chen

ASME Journal Title: Journal of Mechanical Design

Volume/Issue _volume 142, Issue 3, March $2020 \quad$ Date of Publication (VOR* Online) November 1, 2019

https://asmedigitalcollection.asme.org/mechanicaldesign/article/142/3/03

Dynamical-Modeling-and-Control-of-Modular-Snake

ASME Digital Collection URL:

DOI: $\quad$ https://doi.org/10.1115/1.4044691

Repository copy: CC-BY distribution license 


\title{
Dynamical Modelling and Control of Modular Snake Robots with Series Elastic Actuators for Pedal Wave Locomotion on Uneven Terrain
}

\author{
Mohammadali Javaheri Koopaee* \\ Mechanical Engineering Department, \\ University of Canterbury, Christchurch, Canterbury 8041, New Zealand. \\ mohammadali.javaherikoopaee@pg.canterbury.ac.nz

\section{Christopher Pretty} \\ Mechanical Engineering Department, \\ University of Canterbury, Christchurch, Canterbury 8041, New Zealand. \\ chris.pretty@canterbury.ac.nz
}

\section{Koen Classens}

Electrical Engineering Department, Eindhoven University of Technology, 5612 AZ Eindhoven, The Netherlands.

k.h.j.classens@student.tue.nl

\section{XiaoQi Chen}

Manufacturing Futures Research Institute, Swinburne University of Technology, Hawthorn 3122, Australia.

xiaoqichen@swin.edu.au

\section{ABSTRACT}

This paper introduces the equations of motion of modular $2 D$ snake robots moving in vertical plane employing Series Elastic Actuators (SEAs). The kinematics of such 2D modular snake robot is presented in an efficient matrix form and Euler-Lagrange equations have been constructed to model the robot. Moreover, using a spring-damper contact model, external contact forces, necessary for modelling pedal wave motion (undulation in the vertical plane) are taken into account, which unlike existing methods

\footnotetext{
* Corresponding author contact details: E513, Civil Mechanical building, 69 Creyke Road, University of Canterbury, Christchurch, New Zealand. Phone: $+67226741500 ;+6433692393$.

Email: mohammadali.javaherikoopaee@pg.canterbury.ac.nz; m.ali.javaheri@gmail.com.
} 
can be used to model the effect of multiple contact points. Using such contact model, pedal wave motion of the robot has been simulated and the torque signal measured by the elastic element from the simulation and experimentation have been used to show the validity of the model. Moreover, pedal wave locomotion of such robot on uneven terrain is also modelled and an adaptive controller based on torque feedback in gait parameter's space with optimized control gain is proposed. The simulation and experimentation results showed the efficacy of the proposed controller as the robot successfully climbed over a stair-type obstacle without any prior knowledge about its location with at least 24.8 percent higher speed compared to nonadoptive motion.

Keywords: Modular snake robots; equations of motion; pedal wave locomotion; adaptive locomotion; uneven terrain.

\section{INTRODUCTION}

There are many life forms with incredibly effective locomotion mechanisms, sensing and computation capabilities, which are invaluable sources of inspiration for researchers. One of these bio-inspired designs is snake-like robots [1], which their small body cross-section, intrinsic stability, maneuverability and hyper-redundancy make them ideal for locomotion in challenging environments, such as pipes [2], tankers [3] and collapsed buildings [4].

Generally speaking, biological snakes can perform several locomotion patterns, based on environmental conditions, to effectively traverse challenging environments. Among such motion patterns, lateral undulation [5] and its counterpart in the vertical plane (rectilinear [6], or pedal wave motion [7]) are of special interest due to their intrinsic characteristics enabling the snake to move in unstructured environments with different features [8].

Lateral undulation, which is the most common locomotion pattern among biological snakes has received considerable attention since studies on snake robots 
started [9]. Most of these studies consider installing wheels to mimic the anisotropic friction properties of real snakes [10]. However, such wheeled snake robots suffer from the same limitations as wheeled mobile robots have. Hence, they might not be suitable for locomotion in unstructured environments. Moreover, although it has been shown that the anisotropic friction property of the snake belly scales and existence of push points in the environment are the major factors for generating such forward motion in [11], the development of snake-like skin, such as the one proposed in [12], has not yet generated practical results.

To overcome the aforementioned issues, some recent works, such as [13] have focused on designing a robot capable of pushing against the irregularities of the terrain to move forward. Although such obstacle-aided locomotion patterns have been shown to be very effective and do not require the robot to be covered by a snake-like sleeve [14], existence of a sufficient number of pegs for the robot to push against is essential to generate enough propulsive force [15]. Hence, such locomotion mechanisms are effective only for locomotion in confined spaces, such as pipes, where the robot can always push against the walls to move forward [16].

On the other hand, pedal wave motion of the snake robots, which is similar to caterpillar motion [17], is an effective locomotion pattern very similar to lateral undulation but performed in the vertical plane. In this type of motion, the robot lifts some of its links and pushes against the ground to move forward making it very effective for locomotion on uneven terrains, where lateral undulation is no longer effective [7]. Using such a locomotion pattern, one can take advantage of the small cross-section of the snake body to move in confined spaces and because the robot 
pushes against the ground to move forward, existence of sideways push points no longer have an effect on the generation of such movement.

Although pedal wave locomotion patterns can be used in challenging environments, dynamical modelling of such locomotion mechanism, necessary for indepth investigation of such motion, is challenging due to the contact between the robot links and the environment. Some recent works, such as [6] have proposed a modelling framework based on a series of connected masses and springs to model such motion. However, such a model is only suitable for biological snakes modelling, where the contraction and relaxation of the muscles can be modelled with the same method. More recently, in [18] based on the well-known Newton principle and in [19] using the Euler-Lagrange method, the dynamical equations of pedal wave motion suitable for modelling modular snake robots are presented. However, in both of these works, it is assumed that the number of contact points remains constant during the motion, and the normal forces are obtained based on the force and moment balance. Hence, such models are not suitable for modelling a snake robot with multiple contact points in unstructured environments, where the robot might be in contact with multiple contact points in different planes.

In addition to the lack of a generalized modelling framework, the number of works focused on adaptive pedal wave motion on uneven terrain is limited. In [7] a shape-based control scheme has been proposed for the pedal wave locomotion of snakes to enable them to climb a stair-type obstacle. However, the proposed method requires prior information about the size and the location of the obstacle. Moreover, in [20], a control mechanism based on torque control strategy is proposed for a snake- 
like robot. However, the controller requires information from a pressure sensor installed on the surface of the links.

To address the aforementioned issues regarding modelling and control of snake robots with stiff joints on uneven terrain, we proposed a general modeling framework and an adaptive control strategy in [21]. In this work, we introduced the equations of motion of modular 2D snake robots in the vertical plane using the well-known springdamper contact model and proposed an adaptive controller based on external force feedback resulting in the robot to successfully climb over a stair-type obstacle in the simulation environment without experimentation results.

On the other hand, more recently in [22], we proposed a cost-effective snake robot design equipped with Series Elastic Actuators (SEAs), where an elastic element is attached between the motor and the links to reliably measure the servo-motor output torque and regulate the motor torque (force) for adaptive motion [23]. Such a snake robot with SEAs and similar ones such as [24], are less complicated and more robust compared to existing snake robots with torque measurement mechanisms, such as [25] with FSRs (force sensor resistors), [26] with strain gauges and [27] with a cam mechanism (see [22] for more details), which makes them ideal for locomotion on uneven terrain. However, the introduction of an elastic element between the robot servos and the links adds a new level of complexity to the snake robot model [28], which to best of our knowledge is not considered in any snake robot model presented in the literature. Moreover, although it is shown in [29] and [22] (by conducting experimentation on the robot) that adaptive lateral undulation and pedal wave motion can be achieved by incorporating a torque feedback signal (by measuring the 
deflection of the elastic element) into the joint controller, no formal modelling framework exist to be employed for investigation of such control methods.

In this paper, a generalized dynamical model of modular 2D snake robots performing pedal wave locomotion in the vertical plane is presented, which extends our recent work in [21] by taking into account the effect of the flexible element embedded in each joint of the snake robot. The kinematics of such robot has been presented in a matrix form and using the Euler-Lagrange method, the equations of motion of the robot have been derived. Additionally, employing the well-known spring-damper contact model, the effect of external forces on the robot are taken into account, which unlike existing models, such as [19], can be used to model the robot in contact with the environments at multiple points in different planes. Moreover, using this dynamical model, the robot is simulated in an environment with a stair-type obstacle, and an adaptive controller in the gait parameters space with the optimized gain is designed. The effectiveness of such a controller for climbing over an obstacle without prior knowledge about its location is shown in the simulation and experimentation, which shows that at least 24.8 percent faster motion can be achieved with the use of the proposed controller, compared to non-adaptive gaitbased motion generation strategy.

The organization of this paper is as follows. In Section 1, the problem definition is given, the pedal wave motion mechanism of modular snake robots is explained and the design of a snake robot with flexible elements at the joint is briefly discussed. In Section 2, the kinematics of a 2D snake robot with SEAs is presented and in Section 3, the generalized equations of motion of the robot are obtained. Finally, in Section 4, the modelling of the robot in unstructured environments is discussed and an adaptive 
controller based on the servo motor output torque feedback (measured by the elastic element) has been designed. The effectiveness of such a controller is supported by the simulation and experimental results.

\section{BACKGROUND}

Today's world of robotic is completely dominated by wheeled robots. However, this popularity stems from simplicity in modelling, control and manufacture of such robots, and not their capabilities to overcome locomotion challenges. In contrast, snake-like robots have proved to be very effective in unstructured environments, where for wheeled robotic systems, it is impossible to operate without considering additional means of maneuverability [8].

Among snake-like locomotion patterns, pedal wave motion has several advantages over other gaits in unstructured environments. This type of locomotion, which is similar to extended caterpillar locomotion with multiple contact points [17], is exhibited mostly in heavy snakes [30]. Early studies on this type of locomotion suggested that in pedal wave (rectilinear) locomotion snake ribs act as legs, similar to walking. However, the snake does not "travel on its ribs", [31] or in the other words, the snake ribs are not the main means of locomotion.

In pedal wave motion, similar to earthworms [30], the snake travels in a straight line, and unlike other types of locomotion, sideways interaction with the environment is not essential. Instead, contraction and relaxation waves pass over the ventral muscles along with the lifting body parts are the main cause of locomotion [6]. Such motion has some similarities with sidewinding locomotion pattern, which the snake uses two separate parts of the body as static contact points to lift and bend the 
remaining parts and move forward [32]. However, unlike in sidewinding motion, where the robot is moving sidewise, in pedal wave motion the robot moves along a straight line, which makes it more suitable for locomotion in confined spaces, where there is not enough space to move sideways.

To mimic pedal wave motion, different snake robot designs, such as the one presented in [33] are proposed. However, the most common snake-like mechanism, capable of performing pedal wave motion is presented in [22] (see Fig. 1), where a snake robot with six links and five servos rotating about parallel axes has been developed. This robot is capable of generating pedal wave motion by controlling the joints angles based on the following gait pattern, inspired by the body shape of biological snakes:

$$
\alpha_{j}^{d}(t)=A_{p} \sin (\omega t+\eta(j-1))
$$

where $\alpha_{j}^{d} ; j=1,2, \ldots, N-1$ are the desired relative joint angles, $\omega$ is the temporal frequency, $\eta$ is the spatial frequency (phase shift) and $A_{p}$ is the amplitude of the sinusoidal wave. Fig. 1 shows the snake robot with six links performing this type of motion with $A_{p}=\frac{\pi}{4} \mathrm{rad}, \omega=\pi \mathrm{rad} / \mathrm{sec}$ and $\eta=\frac{-2.2 \pi}{5} \mathrm{rad}$ with the average velocity obtained to be $2.40 \mathrm{~cm} / \mathrm{s}$.

In rectilinear motion of biological snakes, the amplitude of the vertical wave, i.e. $A_{p}$ propagating along the snake body is very small, hence such motion is usually slow and not very efficient [34]. However, in a snake robot the maximum amplitude of such vertical waves is not as limited as in biological snakes. Hence, a snake robot can achieve faster motion with the use of such locomotion pattern. 
Moreover, one of the most important characteristics of pedal wave motion is that the snake can lift its body part and push against the ground to move forward, resulting in a very suitable locomotion pattern for uneven terrain. However, this also makes modelling of such motion challenging, compared to lateral undulation, in which the robot body is constantly in contact with the ground [35]. Consequently, any promising dynamical model of pedal wave motion should deal with contact forces in an efficient manner to obtain a suitable simulation model to be used for investigating such a complex motion.

In addition to the complexity of modelling the contact forces, the snake robot joint as shown in Fig. 2, is equipped with an embedded elastic element to reliably measure the motor torque. However, the effect of the elastic element on the robot motion as also mentioned in [36], cannot be neglected and should be considered to obtain a reliable model of the robot.

In the next section, we will mainly focus on obtaining the kinematics of the snake robot with SEAs in XZ plane in a matrix form to be used for obtaining the EulerLagrange equations of motion of the robot.

\section{KINEMATICS OF THE SNAKE ROBOT}

A modular snake robot with $N$ identical links $\left(l_{i} ; i=1,2, \ldots, N\right)$ and $N-1$ actuators $\left(r_{j} ; j=1,2, \ldots, N-1\right)$ with same gear ratio of $n$ attached in series with a spring to the corresponding link, is illustrated in Fig. 3, where $\theta_{i}^{l}$ and $\theta_{j}^{r}$ are the absolute link and rotor angles, respectively, $\alpha_{j}=\theta_{j}^{l}-\theta_{j+1}^{l}$ is the angle between the consecutive links $j$ and $j+1, \beta_{j}=\theta_{j}^{l}-\theta_{j}^{r}$ is the $j^{\text {th }}$ rotor angle relative to its stator reflected through 
the link side, $\left[x_{i}^{l}, z_{i}^{l}\right]^{\mathrm{T}}$ and $\left[x_{j}^{r}, z_{j}^{r}\right]^{\mathrm{T}}$ are the position of the centre of mass of link $l_{i}$ and rotor $r_{j}$, respectively in the global coordinate frame and $\left[p_{x}, p_{z}\right]^{\mathrm{T}}$ denotes the centre of mass of the robot.

It should be noted that rotor angles and the flexible elements at each joint are not shown in Fig. 3 . The reason is that considering the relative link angles $\alpha_{j}$, the body shape of the robot is independent of rotor angles $\theta_{j}^{r}$, hence only relative angles $\alpha_{j}$ s are used to show the body shape of the robot in the vertical plane. Fig. 4 better shows the defined parameters for a single joint of a snake robot equipped with SEAs.

To obtain the expression for the kinematics and dynamics of the snake robot in a matrix form, it is convenient to define several matrices and vectors as follows:

$$
\begin{gathered}
\boldsymbol{H}^{l}=\left[\begin{array}{ccccc}
l & 0 & 0 & \ldots & 0 \\
2 l & l & 0 & \ldots & 0 \\
2 l & 2 l & l & \ldots & 0 \\
\vdots & \vdots & \vdots & \vdots & \vdots \\
2 l & 2 l & 2 l & \ldots & l
\end{array}\right]_{N \times N}, \quad \mathcal{H}^{r}=\left[\begin{array}{cccccc}
2 l & 0 & 0 & \ldots & 0 & 0 \\
2 l & 2 l & 0 & \ldots & 0 & 0 \\
2 l & 2 l & 2 l & \ldots & 0 & 0 \\
\vdots & \vdots & \vdots & \vdots & \vdots & 0 \\
2 l & 2 l & 2 l & \ldots & 2 l & 0
\end{array}\right]_{N-1 \times N} \\
\boldsymbol{C} \boldsymbol{\theta}=\left[\begin{array}{c}
\cos \left(\theta_{1}^{l}\right) \\
\cos \left(\theta_{2}^{l}\right) \\
\vdots \\
\cos \left(\theta_{N}^{l}\right)
\end{array}\right], \boldsymbol{S \theta}=\left[\begin{array}{c}
\sin \left(\theta_{1}^{l}\right) \\
\sin \left(\theta_{2}^{l}\right) \\
\vdots \\
\sin \left(\theta_{N}^{l}\right)
\end{array}\right], \quad\left[\begin{array}{ccccc}
1 & 1 & 1 & \ldots & 1 \\
0 & 1 & 1 & \ldots & 1 \\
0 & 0 & 1 & \ldots & 1 \\
\vdots & \vdots & \vdots & \vdots & \vdots \\
0 & 0 & 0 & \ldots & 0
\end{array}\right]_{N \times N-1} \\
\mathcal{S}_{\theta}=\operatorname{diag}(\boldsymbol{S} \boldsymbol{\theta})=\operatorname{diag}\left(\sin \left(\theta_{1}^{l}\right), \sin \left(\theta_{2}^{l}\right), \ldots, \sin \left(\theta_{N}^{l}\right)\right), \\
\mathcal{C}_{\theta}=\operatorname{diag}(\boldsymbol{C} \boldsymbol{\theta})=\operatorname{diag}\left(\cos \left(\theta_{1}^{l}\right), \cos \left(\theta_{2}^{l}\right), \ldots, \cos \left(\theta_{N}^{l}\right)\right),
\end{gathered}
$$

where $O_{N \times(N-1)}$ is an $N \times(N-1)$ null matrix with the specified dimension. Considering these matrices, the kinematics relation for the rotors and the links can be obtained in a matrix form, as will be discussed in following sections. 


\section{The robot links velocity}

Considering the body shape of the robot in Fig. 3 and assuming that $l_{1}=l_{2}=$ $\cdots=l_{N}=2 l$ for simplicity, the position of centre of mass of each link can be derived as the function of absolute joint angles as follows:

$$
\begin{aligned}
& x_{i}^{l}=x_{0}+2 l \sum_{k=1}^{i-1} \cos \left(\theta_{k}^{l}\right)+l \cos \left(\theta_{i}^{l}\right) \\
& z_{i}^{l}=z_{0}+2 l \sum_{k=1}^{i-1} \sin \left(\theta_{k}^{l}\right)+l \sin \left(\theta_{i}^{l}\right) .
\end{aligned}
$$

which with little effort can be written in matrix form as below:

$$
\begin{aligned}
\boldsymbol{X}^{l} & =\boldsymbol{V}^{l} x_{0}+\mathcal{H}^{l} \boldsymbol{C} \boldsymbol{\theta}, \\
\boldsymbol{Z}^{l} & =\boldsymbol{V}^{l} z_{0}+\mathcal{H}^{l} \boldsymbol{S} \boldsymbol{\theta}
\end{aligned}
$$

where $\boldsymbol{X}^{\boldsymbol{l}}=\left[\begin{array}{llll}x_{1}^{l} & x_{2}^{l} & \ldots & x_{N}^{l}\end{array}\right]^{\mathrm{T}}, \boldsymbol{Z}^{\boldsymbol{l}}=\left[\begin{array}{llll}z_{1}^{l} & z_{2}^{l} & \ldots & z_{N}^{l}\end{array}\right]^{\mathrm{T}}$ and $\boldsymbol{V}^{l}=\left[\begin{array}{llll}1 & 1 & \ldots & 1\end{array}\right]_{N \times 1}^{\mathrm{T}}$. Considering that $\left[p_{x}, p_{z}\right]^{\mathrm{T}}=1 / N\left[\sum_{i=1}^{N} x_{i}^{l}, \sum_{i=1}^{N} z_{i}^{l}\right]^{\mathrm{T}}$, and replacing $x_{i}$ and $z_{i}$ from (2) and ( 3 ) the velocity of centre of mass of each link can be obtained to be:

$$
\begin{aligned}
& \dot{\boldsymbol{X}}^{l}=\boldsymbol{V}^{l} \dot{p}_{x}+\frac{1}{N} \boldsymbol{V}^{l}\left(\boldsymbol{V}^{l}\right)^{T} \mathcal{H}^{l} \mathcal{S}_{\theta} \dot{\boldsymbol{\theta}}^{l}-\mathcal{H}^{l} \mathcal{S}_{\theta} \dot{\boldsymbol{\theta}}^{l} \\
& \dot{\boldsymbol{Z}}^{l}=\boldsymbol{V}^{l} \dot{p}_{z}-\frac{1}{N} \boldsymbol{V}^{l}\left(\boldsymbol{V}^{l}\right)^{T} \mathcal{H}^{l} \mathcal{C}_{\theta} \dot{\boldsymbol{\theta}}^{l}+\mathcal{H}^{l} \mathcal{C}_{\theta} \dot{\boldsymbol{\theta}}^{l},
\end{aligned}
$$

where $\dot{\boldsymbol{\theta}}^{l}=\left[\begin{array}{llll}\dot{\theta}_{1}^{l} & \dot{\theta}_{2}^{l} & \ldots & \dot{\theta}_{N}^{l}\end{array}\right]^{\mathrm{T}}$ is the vector of angular velocities of the links and $\dot{p}_{x}$ and $\dot{p}_{z}$ are the velocity of the centre of mass of the robot.

Noting that $\theta_{i}^{l}=\sum_{k=i}^{N-1}\left(\alpha_{k}\right)+\theta_{N}^{l}$ and defining the generalized coordinates to be $\boldsymbol{q}=\left[\alpha_{1}, \ldots, \alpha_{N-1}, \theta_{N}^{l}, p_{x}, p_{z}, \beta_{1}, \ldots, \beta_{N-1}\right]^{\mathrm{T}}$, it is possible to represent the kinematic equations of the links as a function of $\boldsymbol{q}$ and $\dot{\boldsymbol{q}}$ as below: 


$$
\begin{gathered}
\dot{\boldsymbol{X}}^{l}=\left[\begin{array}{cc}
\left(\mathcal{B}_{1}^{l}+\mathcal{A}^{l} \mathcal{S}_{\theta} \mathcal{C}\right) & \mathcal{O}_{l}
\end{array}\right] \dot{\boldsymbol{q}}, \\
\dot{\boldsymbol{Z}}^{l}=\left[\begin{array}{cc}
\left(\mathcal{B}_{2}^{l}-\mathcal{A}^{l} \mathcal{C}_{\theta} \mathcal{C}\right) & \mathcal{O}_{l}
\end{array}\right] \dot{\boldsymbol{q}} \\
\dot{\boldsymbol{\theta}}^{l}=\left[\begin{array}{ll}
\mathcal{C} & \mathcal{O}_{l}
\end{array}\right] \dot{\boldsymbol{q}},
\end{gathered}
$$

where $\mathcal{B}_{1}^{l}=\left[\begin{array}{lll}O_{N \times N} & V^{l} & 0_{N \times 1}\end{array}\right], \mathcal{B}_{2}^{l}=\left[\begin{array}{ll}O_{N \times(N+1)} & \boldsymbol{V}^{l}\end{array}\right], \mathcal{A}^{l}=-\mathcal{H}^{l}+\frac{1}{N} \boldsymbol{V}^{l}\left(\boldsymbol{V}^{l}\right)^{\mathrm{T}} \mathcal{H}^{l}$, $\mathcal{C}=\left[\begin{array}{lll}\mathcal{F} & V^{l} & O_{N \times 2}\end{array}\right]$ and $O_{N \times N}$ is a matrix with all zero elements.

\section{The robot rotors velocity}

In addition to the linear velocity of the centre of mass and the angular velocity of each link, the angular velocity of the rotors and the linear velocity of the centre of mass of the rotors should also be obtained to calculate the expression for the total kinetic energy of the system. Considering the body shape of the robot in Fig. 3, the position of the centre of mass of rotor $r_{j}$ can immediately be obtained via:

$$
\begin{aligned}
& x_{j}^{r}=x_{0}+2 l \sum_{k=1}^{j} \cos \left(\theta_{k}^{l}\right) \\
& z_{j}^{r}=z_{0}+2 l \sum_{k=1}^{j} \sin \left(\theta_{k}^{l}\right),
\end{aligned}
$$

which using a similar method as the previous section can be written as:

$$
\begin{gathered}
\boldsymbol{X}^{r}=\boldsymbol{V}^{r} x_{0}+\mathcal{H}^{r} \boldsymbol{C} \boldsymbol{\theta}, \\
\boldsymbol{Z}^{r}=\boldsymbol{V}^{r} z_{0}+\mathcal{H}^{r} \boldsymbol{S} \boldsymbol{\theta}
\end{gathered}
$$

where $\boldsymbol{X}^{\boldsymbol{r}}=\left[\begin{array}{llll}x_{1}^{r} & x_{2}^{r} & \ldots & x_{N-1}^{r}\end{array}\right]^{\mathrm{T}}, \boldsymbol{Z}^{r}=\left[\begin{array}{llll}z_{1}^{r} & z_{2}^{r} & \ldots & z_{N-1}^{r}\end{array}\right]^{\mathrm{T}} \quad$ and $\quad \boldsymbol{V}^{r}=$ $\left[\begin{array}{llll}1 & 1 & \ldots & 1\end{array}\right]_{(N-1) \times 1}^{\mathrm{T}}$. Obtaining the derivate of the vector $\boldsymbol{X}^{r}$ and $\boldsymbol{Z}^{r}$ with respect to time, the velocity of centre of mass of each rotor can be obtained to be: 


$$
\begin{aligned}
& \dot{\boldsymbol{X}}^{r}=\left[\begin{array}{ll}
\left(\mathcal{B}_{1}^{r}+\mathcal{A}^{r} \mathcal{S}_{\theta} \mathcal{C}\right) & \mathcal{O}_{r}
\end{array}\right] \dot{\boldsymbol{q}}, \\
& \dot{\boldsymbol{Z}}^{r}=\left[\begin{array}{ll}
\left(\mathcal{B}_{2}^{r}-\mathcal{A}^{r} \mathcal{C}_{\theta} \mathcal{C}\right) & \mathcal{O}_{r}
\end{array}\right] \dot{\boldsymbol{q}}, \\
& \dot{\boldsymbol{\theta}}^{r}=\mathcal{L} \dot{\boldsymbol{q}}
\end{aligned}
$$

where $\mathcal{B}_{1}^{r}=\left[\begin{array}{lll}O_{(N-1) \times N} & \boldsymbol{V}^{r} & O_{(N-1) \times 1}\end{array}\right], \mathcal{B}_{2}^{r}=\left[O_{(N-1) \times(N+1)} \quad \boldsymbol{V}^{r}\right], \mathcal{A}^{r}=-\mathcal{H}^{r}+$ $\frac{1}{N} \boldsymbol{V}^{r}\left(\boldsymbol{V}^{l}\right)^{\mathrm{T}} \mathcal{H}^{l}, \quad \mathcal{L}=\left[\begin{array}{ll}\mathcal{C}_{1} & -\mathcal{P}\end{array}\right], \mathcal{C}=\left[\begin{array}{c}\mathcal{C}_{1(N-1) \times(N+2)} \\ \mathcal{C}_{21 \times(N+2)}\end{array}\right]$ and $\mathcal{P}$ is a diagonal matrix containing the identical gear ratio of the $N-1$ motors.

It should be noted that the presented procedure for obtaining the kinematics of the links and the rotors, enabled us to obtain the position and velocity of the centre of mass of each link and rotor as a function of joint angles $\alpha_{i}$, rotor angle $\beta_{j}$, the absolute angle of the head module and the position of centre of mass of the robot in matrix form. Hence the implementation of such equations does not require symbolic computation, making the implementation of equations of motion to be presented in the next section simpler. It should also be mentioned that although the kinematic relations obtained for the snake robot in the vertical plane $\mathrm{XZ}$, the same equations can describe the kinematic relations of lateral undulation locomotion in horizontal plane $X Y$ by only replacing $Z$ axis with $Y$ axis. Hence, the obtained relations can be used to describe the kinematics of any modular 2D snake robot with an arbitrary number of links in an easy to implement matrix form.

\section{DYNAMICAL EQUATIONS}

Considering the number of degrees of freedom of the snake robot, the EulerLagrange method is a straightforward approach, which can be employed to obtain the equations of motion of the snake robot. Using this method, the external contact forces Javaheri Koopaee

MD-19-1451 
can be incorporated into the equations, the effect of the springs at each joint can be taken into account through the expression for the potential energy of the system and the gravitational forces can easily be handled. Thus, compared to Newton's method, Euler-Lagrange method can be used to derive the dynamical equations of the snake robot motion in the vertical plane with less complexity.

Choosing generalized coordinates, i.e. the minimum number of variables required to uniquely describe the system, to be $\boldsymbol{q}$, the Euler-Lagrange equations of motion can immediately be constructed as follows:

$$
\frac{d}{d t}\left(\frac{\partial\left(T_{1}+T_{2}\right)}{\partial \dot{\boldsymbol{q}}}\right)-\frac{\partial\left(T_{1}+T_{2}\right)}{\partial \boldsymbol{q}}+\frac{\partial\left(V_{1}+V_{2}+V_{3}\right)}{\partial \boldsymbol{q}}=\boldsymbol{B}+\boldsymbol{Q}^{c}
$$

where $T_{1}$ and $T_{2}$ are the kinetic energy of the links and the rotors respectively, $V_{1}$ is the potential energy stored in the flexible elements (torsional spring) at the joints, $V_{2}$ and $V_{3}$ are the potential energy of the links and the rotors due to the gravitational force, $\boldsymbol{B}=\left[\begin{array}{c}\mathbf{0}_{f} \\ \boldsymbol{U}\end{array}\right], \quad \boldsymbol{O}_{f}$ is a zero column vector with $(N+2)$ rows, $\boldsymbol{U}$ is the vector of $N-1$ control inputs (motor torques) and $\boldsymbol{Q}^{c}$ is the vector of non-conservative contact forces.

\section{Kinetic Energy}

Considering the chosen generalized coordinates $\boldsymbol{q}$, it can be seen that the expression for the kinetic energy of the links, necessary for constructing the EulerLagrange equation is independent of rotor angles $\beta_{i}$ s, i.e. the body shape of the robot is independent of $\beta_{i}$. Hence, $T_{1}$ can be obtained to be only the sum of the kinetic energy of the links due to their own linear and angular velocities as follows: 


$$
T_{1}=\frac{1}{2}\left(\dot{\boldsymbol{X}}^{l}\right)^{T} \mathcal{M}^{l}\left(\dot{\boldsymbol{X}}^{l}\right)+\frac{1}{2}\left(\dot{\boldsymbol{Z}}^{l}\right)^{T} \mathcal{M}^{l}\left(\dot{\boldsymbol{Z}}^{l}\right)+\frac{1}{2}\left(\dot{\boldsymbol{\theta}}^{l}\right)^{T} \mathcal{J}^{l}\left(\dot{\boldsymbol{\theta}}^{l}\right)
$$

where $\mathcal{M}^{l}$ and $\mathcal{J}^{l}$ are $N$ by $N$ diagonal matrices of mass and moment of inertial of the links respectively. It is worthwhile to mention that, in case of a 2D snake robot with stiff joints, the kinetic energy of the links as presented in (19) will be the only terms required to calculate the kinetic energy of the system and obtain the equations of motion of the robot. The only consideration is that the mass of each link should be the sum of the mass of the rotors and the links.

To fully obtain the expression for the kinetic energy of the system, the kinetic energy of the rotors should also be taken into account. Similar to the method used in the previous section, the kinetic energy of the rotors can be obtained as:

$$
T_{2}=\frac{1}{2}\left(\dot{\boldsymbol{X}}^{r}\right)^{\mathrm{T}} \mathcal{M}^{r}\left(\dot{\boldsymbol{X}}^{r}\right)+\frac{1}{2}\left(\dot{\boldsymbol{Z}}^{r}\right)^{\mathrm{T}} \mathcal{M}^{r}\left(\mathrm{Z}^{r}\right)+\frac{1}{2}\left(\dot{\boldsymbol{\theta}}^{r}\right)^{\mathrm{T}} \mathcal{J}^{r}\left(\dot{\boldsymbol{\theta}}^{r}\right)
$$

where $\dot{\boldsymbol{X}}^{r}$ and $\dot{\boldsymbol{Z}}^{r}$ are the linear velocities of the rotors at each joint, independent of the rotor angle $\beta_{j}, \dot{\boldsymbol{\theta}}^{r}$ is the angular velocity of the rotor and $\mathcal{M}^{r}$ and $\mathcal{J}^{r}$ are $(N-1)$ by $(N-1)$ diagonal matrices of mass and moment of inertia of the rotors.

\section{Potential Energy}

The first part of the potential energy of the snake robot as shown in Fig. 4 is due to the springs attached between the actuators and links. Hence, the potential energy of the system due to the torsional spring at the joints can be obtained as:

$$
V_{1}=\frac{1}{2}(\boldsymbol{\beta}-\boldsymbol{\alpha})^{\mathrm{T}} K(\boldsymbol{\beta}-\boldsymbol{\alpha}),
$$


where $K$ is the $N-1$ by $N-1$ diagonal matrix containing stiffness of the flexible elements of the joint, $\boldsymbol{\alpha}=\left[\alpha_{1}, \ldots, \alpha_{N-1}\right]^{\mathrm{T}}$ and $\boldsymbol{\beta}=\left[\beta_{1}, \ldots, \beta_{N-1}\right]^{\mathrm{T}}$. This expression can also be represented as a function of generalized coordinates as follows:

$$
V_{1}=\frac{1}{2} \boldsymbol{q}^{\mathrm{T}} K_{T} \boldsymbol{q}
$$

where $K_{T}$ is defined to be:

$$
K_{T}=\left[\begin{array}{ccc}
K_{(N-1) \times(N-1)} & O_{(N-1) \times 3} & -K_{(N-1) \times(N-1)} \\
O_{3 \times(N-1)} & O_{3 \times 3} & O_{3 \times(N-1)} \\
-K_{(N-1) \times(N-1)} & O_{(N-1) \times 3} & K_{(N-1) \times(N-1)}
\end{array}\right]
$$

where $O_{3 \times(N-1)}, O_{(N-1) \times 3}$ and $O_{3 \times 3}$ are null matrices with the specified dimensions.

On the other hand, considering the body shape of the robot in XZ plane, the robot lifts some parts of its body from the ground. Thus, the expression for the potential energy of the system due to the mass of the robot should also be included. Considering the body shape of the robot the expression for the potential energy of the system due to the mass of the links $\left(V_{2}\right)$ can be obtained to be:

$$
g\left(\boldsymbol{V}^{l}\right)^{\mathrm{T}} \mathcal{M}^{l} \times \boldsymbol{Z}^{l}=\mathrm{g}\left(\boldsymbol{V}^{l}\right)^{\mathrm{T}} \mathcal{M}^{l} \times \boldsymbol{V}^{l} z_{0}+\mathcal{H}^{l} \boldsymbol{S} \boldsymbol{\theta}
$$

Knowing that $p_{z}=1 / N \sum_{i=1}^{N} z_{i}^{l}$ and substituting $z_{0}$ from the expression of centre of mass of the robot, (24) can be written as follows:

$$
V_{2}=g\left(\boldsymbol{V}^{l}\right)^{T} \mathcal{M}^{l} \boldsymbol{Z}^{l}=g\left(\boldsymbol{V}^{l}\right)^{T} \mathcal{M}^{l}\left(\mathcal{B}_{2}^{l}-\mathcal{A}^{l} \mathcal{C}_{\theta} \mathcal{C}\right) \boldsymbol{q}
$$

Moreover, the centre of mass of the rotors are located at $\left[x_{j}^{r}, z_{j}^{r}\right]$ and not at centre of mass of the links, thus similar procedure should be repeated to calculate the potential energy of the rotors, which will result in the following expression for the potential energy of the rotors: 


$$
V_{3}=\mathrm{g}\left(\boldsymbol{V}^{r}\right)^{\mathrm{T}} \mathcal{M}^{r} \boldsymbol{Z}^{r}=\mathrm{g}\left(\boldsymbol{V}^{r}\right)^{\mathrm{T}} \mathcal{M}^{r}\left(\mathcal{B}_{2}^{r}-\mathcal{A}^{r} \mathcal{C}_{\theta} \mathcal{C}\right) \boldsymbol{q}
$$

Hence one can find the total potential energy of the system to be the sum of the potential energy due to the mass of the links, mass of the rotors and the elastic elements of the joint.

\section{Non-Conservative Forces}

To obtain the effect of non-conservative forces $f_{i}^{x}, f_{i}^{Z}$ on the $i^{\text {th }}$ link of the robot expressed in the global coordinate frame, it is enough to consider the point of effect of such forces. Assuming that these forces will be exerted on the centre of mass of each link, $\boldsymbol{Q}^{c}$ can be obtained as follows:

$$
\boldsymbol{Q}^{c}=\sum_{i=1}^{N}\left[\begin{array}{ll}
\frac{\partial \dot{x}_{i}^{l}}{\partial \dot{\boldsymbol{q}}} & \frac{\partial \dot{z}_{i}^{l}}{\partial \dot{\boldsymbol{q}}}
\end{array}\right]^{T}\left[\begin{array}{l}
f_{i}^{x} \\
f_{i}^{Z}
\end{array}\right]
$$

which can be written in matrix form as:

$$
\boldsymbol{Q}^{c}=\left[\begin{array}{ll}
\frac{\partial \dot{\boldsymbol{X}}^{l}}{\partial \dot{\boldsymbol{q}}} & \frac{\partial \dot{\boldsymbol{Z}}^{l}}{\partial \dot{\boldsymbol{q}}}
\end{array}\right]\left[\begin{array}{l}
\boldsymbol{F}^{x} \\
\boldsymbol{F}^{z}
\end{array}\right]
$$

where $\frac{\partial \dot{X}^{l}}{\partial \dot{\boldsymbol{q}}}=\left[\left(\mathcal{B}_{1}^{l}+\mathcal{A}^{l} \mathcal{S}_{\theta} \mathcal{C}\right) \quad \mathcal{O}_{l}\right]^{\mathrm{T}}, \quad \frac{\partial \dot{Z}^{l}}{\partial \dot{\boldsymbol{q}}}=\left[\begin{array}{lll}\left(\mathcal{B}_{2}^{l}-\mathcal{A}^{l} \mathcal{C}_{\theta} \mathcal{C}\right) & \mathcal{O}_{l}\end{array}\right]^{\mathrm{T}} \quad$ and $\quad \boldsymbol{F}^{x}=$ $\left[\begin{array}{llll}f_{x 1} & f_{x 2} & \ldots & f_{x N}\end{array}\right]^{T}, \boldsymbol{F}^{z}=\left[\begin{array}{lllll}f_{z 1} & f_{z 2} & \ldots & f_{z N}\end{array}\right]^{T}$ are the vectors of all nonconservative forces along the global $\mathrm{X}$ and $\mathrm{Z}$ direction respectively.

The above procedure can be used to take into account any non-conservative forces such as friction and/or contact forces, necessary to model both pedal wave motion and lateral undulation in confined spaces. For example, Fig. 5 shows a general case where a single link of the robot is in contact with an obstacle and $f_{k}^{N}$ and $f_{k}^{T}$ are the normal and tangential forces exerted on the robot at the point $p_{k}$ by the environment. 
Assuming that $p_{k}$ is in contact with the obstacle, i.e. $z^{p_{k}} \leq 0$, and considering a spring-damper contact model, $f_{k}^{N}$ can be calculated as follows:

$$
f_{k}^{N}=\operatorname{Max}\left(k\left(z^{p_{k}}\right)-d \dot{z}^{\left.p_{k}, 0\right)}\right.
$$

where $Z^{p_{k}}$ is the coordinate of the point of contact $p_{k}$ along the $Z^{\prime}$ ( $z$ axis of the attached coordinate $O X^{\prime} Z^{\prime}$ to the obstacle), $k$ is the spring and $d$ is the damping constant of the environment. Once $f_{k}^{N}$ is obtained, it is straightforward to calculate $f_{k}^{T}$ as follows:

$$
f_{k}^{T}=-f_{k}^{N} \mu_{C} \operatorname{sign}\left(\dot{x}^{p_{k}}\right)
$$

where $\dot{x}^{p_{k}}$ is the velocity of the point $p_{k}$ along the direction tangent to the surface and $\mu_{C}$ is the friction coefficient.

Having derived $f_{k}^{N}$ and $f_{k}^{T}$, it will be straight forward to incorporate these forces into the equations of motion. However, as it is shown in the general case of Fig. $5, f_{k}^{N}$ and $f_{k}^{T}$ are expressed in the stationary coordinate frame $O X^{\prime} Z^{\prime}$, which is not necessarily aligned with the global coordinate frame. Thus, firstly, such forces should be expressed in the global coordinate frame to construct $\boldsymbol{F}_{x}$ and $\boldsymbol{F}_{Z}$ and then equation (28) should be used to incorporate these forces into the dynamical model.

Another important consideration about such contact model for the snake robot is that unlike friction forces, which are assumed to be exerted on the centre of mass of each link, the contact forces might be exerted on any point located on the robot links. However, due to computational limitations, it is not possible to check every points on the robot links for a possible contact with the environment. Hence, for the simulation purpose, we only consider the centre of mass of the links, robot joints, tip of the head and the tail to be checked at each step-time to calculate the contact forces. 
This means that for a snake robot with $N$ links and $N-1$ joints, $2 N+1$ points on the robot should be tested at each time-step to see if a contact has been occurred or not. Once the set of contact points, i.e. $p_{k} ; k=1, \ldots, K$ are obtained, the tangential and normal forces at these points can be calculated based on (29) and (30). It is critical to mention that, to incorporate these forces into the equations of motion, the derivative of the velocity vector of the contact points with respect to $\boldsymbol{q}$ needs to be obtained and then a similar relation as in (28) should be used, which is straight forward and not included here for the sake of brevity.

\section{Equations of Motion}

Considering the expression obtained for the kinetic and potential energies of the snake robot, it is now possible to construct the equations of motion of pedal wave motion as follows:

$$
\begin{array}{r}
{\left[\begin{array}{cc}
M^{L}\left(\boldsymbol{q}_{\mathbf{1}}\right)+M^{R}\left(\boldsymbol{q}_{\mathbf{1}}\right)+S_{1} & S_{2} \\
S_{2}^{\mathrm{T}} & S_{3}
\end{array}\right] \ddot{\boldsymbol{q}}+\left[\begin{array}{c}
\boldsymbol{C}^{L}\left(\boldsymbol{q}_{\mathbf{1}}, \dot{\boldsymbol{q}}_{\mathbf{1}}\right)+\boldsymbol{C}^{R}\left(\boldsymbol{q}_{\mathbf{1}}, \dot{\boldsymbol{q}}_{\mathbf{1}}\right) \\
\mathbf{0}_{\boldsymbol{A}}
\end{array}\right]} \\
+K_{T} \boldsymbol{q}+\boldsymbol{g}=\boldsymbol{B}+\boldsymbol{Q}^{c}
\end{array}
$$

where $M^{L}\left(\boldsymbol{q}_{\mathbf{1}}\right)$ is a $(N+2) \times(N+2)$ positive definite link inertia matrix, $M^{R}\left(\boldsymbol{q}_{\mathbf{1}}\right)$ is the rotor inertia matrix, $\boldsymbol{C}^{L}\left(\boldsymbol{q}_{\mathbf{1}}, \dot{\boldsymbol{q}}_{\mathbf{1}}\right)$ and $\boldsymbol{C}^{R}\left(\boldsymbol{q}_{\mathbf{1}}, \boldsymbol{q}_{\mathbf{1}}\right)$ are Coriolis and centrifugal terms due to the velocity of the links and rotors, $\mathbf{0}_{\boldsymbol{A}}$ is a zero vector of size $N-1, \mathcal{G}$ is the column vector of gravitational forces, $\boldsymbol{B}$ is the same as defined in (18) and

$$
\left[\begin{array}{ll}
S_{1} & S_{2} \\
S_{2}^{T} & S_{3}
\end{array}\right]=\mathcal{L}^{T} \mathcal{J}^{r} \mathcal{L}
$$

where $S_{1}$ and $S_{2}$ appeared due to the angular velocity of the rotors depending on $\dot{\boldsymbol{\theta}}^{l}$ and $S_{3}$ depends on the inertia and gear ratio of the rotors. (See the Appendix for the structure of the matrices in (31)). 
Equation (31) can be seen as two sets of equations. The first $N+2$ equations are under-actuated dynamics of the system containing the relative joint angles, the position of the centre of mass and orientation of the robots head module, in which the friction force and other environmental forces will appear and the last $N-1$ equations are fully actuated motor-side equations.

It should also be noted that considering stiff joints, i.e. $K$ matrix with high values, one can assume there is no elastic element at the joint, i.e. the last $N-1$ equations can be neglected. Consequently, the equations of motion of a planar snake robot with stiff joints can easily be obtained with little effort as follows:

$$
\begin{aligned}
\left(M^{L}\left(\boldsymbol{q}_{1}\right)+M^{R}\left(\boldsymbol{q}_{\mathbf{1}}\right)+S_{1}\right) \ddot{\boldsymbol{q}}_{\mathbf{1}} & +\boldsymbol{C}^{L}\left(\boldsymbol{q}_{\mathbf{1}}, \dot{\boldsymbol{q}}_{\mathbf{1}}\right) \\
& +\boldsymbol{C}^{\boldsymbol{R}}\left(\boldsymbol{q}_{\mathbf{1}}, \dot{\boldsymbol{q}}_{\mathbf{1}}\right)+\boldsymbol{G}^{\prime}=\boldsymbol{B}^{\prime}+\boldsymbol{Q}^{c^{\prime}}
\end{aligned}
$$

where the definition of $M^{L}\left(\boldsymbol{q}_{1}\right), M^{R}\left(\boldsymbol{q}_{1}\right), \boldsymbol{C}^{L}\left(\boldsymbol{q}_{\mathbf{1}}, \dot{\boldsymbol{q}}_{\mathbf{1}}\right)$ and $\boldsymbol{C}^{R}\left(\boldsymbol{q}_{\mathbf{1}}, \dot{\boldsymbol{q}}_{\mathbf{1}}\right)$ remain to be the same as (31), $\boldsymbol{B}^{\prime}=\left[\begin{array}{c}\boldsymbol{U} \\ \mathbf{0}_{\boldsymbol{s}}\end{array}\right], \mathbf{0}_{\boldsymbol{s}}$ is a zero vector with three elements, and $\boldsymbol{q}_{\mathbf{1}}, \boldsymbol{G}^{\prime}$ and $\boldsymbol{Q}^{c^{\prime}}$ are first $N+2$ elements of $\boldsymbol{q}, \boldsymbol{G}$, and $\boldsymbol{Q}^{c}$, respectively.

\section{PEDAL WAVE MOTION ON SMOOTH SURFACES}

To demonstrate the effectiveness of the proposed model in generating pedal

wave motion, gait pattern (1) with $A_{p}=\frac{\pi}{4} \mathrm{rad}, \omega=\pi \mathrm{rad} / \mathrm{sec}$ and $\eta=\frac{-2.2 \pi}{2} \mathrm{rad}$ (same as the pedal wave motion of the robot shown in Fig. 1) has been chosen to be implemented on the simulation model (31). For this purpose, a snake robot with six identical links each with the mass of $0.1 \mathrm{~kg}$ and length $0.07 \mathrm{~m}$ (same as the physical robot) and five motors with the rotor mass of $0.02 \mathrm{~kg}$ and gear reduction of 255 has been simulated via MatlabR2017b, and the results demonstrating pedal wave motion Javaheri Koopaee

MD-19-1451 
are shown in Fig. 6. As shown in Fig. 6, the proposed dynamical model has been successfully used to simulate pedal wave motion when the environmental constant $k$ and $d$ are chosen to be $350 \frac{N}{m}$ and $15 \frac{N s e c}{m}$ respectively. The simulation step time has been chosen to be $0.0001 \mathrm{~s}$ which is sufficiently small to make sure the fast dynamic of the system (last $N-1$ rows of (31)) are being captured.

It should be noted that, to implement gait equation (1) a joint level controller should be designed such that the error between the desired and measured relative joint angles converges to zero by only using motor-side measurements $\beta_{j}$ and $\dot{\beta}_{j}$. Generally speaking, designing such a tracking controller for flexible joint robots requires back-stepping control techniques [37]. However, for such small values of $A_{p}$ and $\omega_{1}$ it can be concluded that $\ddot{\alpha}_{j}^{d}$ and $\dddot{\alpha}_{j}^{d}$ will be sufficiently small [38], hence a simple PD tracking controller as presented in (33), is used to control the simulated model in Fig. 6 and also the physical robot.

$$
\boldsymbol{U}=-K_{p}\left(\beta_{j}-\alpha_{j}^{d}\right)-K_{D}\left(\dot{\beta}_{j}-\dot{\alpha}_{j}^{d}\right)
$$

where $K_{p}$ and $K_{D}$ are positive definite gains chosen to be 30 and 15 respectively.

To validate the proposed model in (31), the torque signal obtained by measuring the deflection of the elastic element from the experiment shown in Fig. 1 has been compared with the simulation results for the fifth joint (head module) as shown in Fig. 7. As can be seen in Fig. 7, the experimental data resembles the simulation data with some expected inconsistency due to the simplified assumptions used to obtain model (31). The main differences between the model and the actual robot could stem from the assumed linear model for the elastic element, difficulty in precisely modeling the contact forces and the very simplified friction model (30). 
Moreover, as it can be seen in Fig. 1, at first, the robot is moving on top of a high friction surface (black plate) with $\mu_{C}=0.82$ and then on a low friction surface with $\mu_{C}=0.40$ to examine the effect of friction on the forward velocity of the robot and investigate the relationship between the friction of the surface and the deflection of the elastic element. From the experiment, it could be seen that the forward velocity of the robot on the surface with higher friction was approximately 20.3 percent higher than the robot velocity on the lower friction surface. Considering (30), one may expect that the velocity of the robot on high friction to be almost two times higher than the velocity on the lower friction surface as the external force in $X$ direction linearly depends on $\mu_{C}$. However, both the simulation results (13.7 percent increase) and experimental results suggested that increasing $\mu_{C}$ will cause the forward velocity to increase, but this relation is not linear.

Moreover, as can be seen in Fig. 7, after 7.5s, when the entire head module is on the surface with low friction, the absolute value of the negative peaks of the torque signal have been increased by 16.3 percent. Although in the experimentation, the same phenomenon has been observed, the peaks were increased by only 4.3 percent. One reason for that could be using the very simplified model of friction in (30) for the simulation, which differs from the reality.

Consequently, one can see that, model (31) is successfully used to capture the essentials of pedal wave motion. However, as expected, the simulation model cannot precisely capture the details of pedal wave motion of a real snake robot, due to the complexity of precisely modelling the contact forces and the friction. Hence, such simulation framework combined with real experimentation on the physical robot can generate more fruitful results. 


\section{PEDAL WAVE MOTION ON UNEVEN TERRAIN}

Unlike locomotion on smooth surfaces, on uneven terrains, the environmental forces exerted on the robot along the forward direction might cancel each other. Hence, the robot average velocity could be effected adversely and in some cases, the robot might not be able to move forward (see the video: https://www.youtube.com/watch?v=18j0gGK4wR8). To address this issue, one can extend the presented model in (31) to simulate pedal wave motion on uneven terrains and investigate adaptive control strategies to achieve more agile snake like motion on uneven terrains.

For this purpose, motivated by the controller proposed in [29] for lateral undulation, we propose an adaptive control strategy based on the following extended gait pattern:

$$
\alpha_{j}^{d}\left(t, \eta_{j}\right)=A_{p} \sin \left(\omega t+\eta_{j}(j-1)\right)
$$

where $A_{p}, \omega$ are constant gait parameters similar to (1) and $\eta_{j}$ is the spatial frequency of $j^{\text {th }}$ joint to be controlled.

To control the spatial frequencies, i.e. the vector $\Pi=\left[\eta_{1}, \eta_{2}, \ldots, \eta_{N-1}\right]^{T}$, we propose an adaptive controller shown in Fig. 8 , where $C_{p}$ is the position controller (33), $\boldsymbol{\Pi}^{\boldsymbol{n}}$ is the vector of nominal spatial frequencies, $\boldsymbol{\Phi}^{d}=\left[\alpha_{1}^{d}, \alpha_{2}^{d}, \ldots, \alpha_{N-1}^{d}\right]^{T}$ is the vector of the desired joint angles generated from (34). Moreover, the admittance controller is defined as follows:

$$
M_{d}\left(\ddot{\mathbf{\Pi}}-\ddot{\boldsymbol{\Pi}}^{\mathbf{n}}\right)+D_{d}\left(\dot{\boldsymbol{\Pi}}-\dot{\boldsymbol{\Pi}}^{\mathbf{n}}\right)+K_{d}\left(\boldsymbol{\Pi}-\boldsymbol{\Pi}^{\mathbf{n}}\right)=k_{c} \boldsymbol{Q}^{e}
$$


where $M_{d}, D_{d}$, and $K_{d}$ are the positive desired inertia, damping and stiffness, respectively, $\boldsymbol{Q}^{\boldsymbol{e}}$ is the vector of torque signal feedback measured by $N-1$ elastic elements embedded at each joint (first $N-1$ elements of $K_{T} \boldsymbol{q}$ ) and $k_{\boldsymbol{c}}$ is the feedback gain to be designed.

As it can be seen in (35), choosing the nominal gait parameters $\Pi^{n}$, if $Q^{e} \cong 0$, $\Pi$ converges to $\Pi^{n}$, because $M_{d}, D_{d}$, and $K_{d}$ are chosen to guarantee (35) is a stable second order system. However, if $\boldsymbol{Q}^{e} \neq 0$ (mainly due to external contact forces), $\Pi$ deviates from its nominal value based on the feedback signal $\boldsymbol{Q}^{e}$ and the dynamical model presented in (35). This is similar to the conventional admittance controllers, where the measured output torque (force) will be used in the outer loop to change the reference for the inner position control loop [40]. However, in the proposed controller in (35), the measured torque signal will be used to adaptively control the parameter of the gait pattern, i.e. $\eta_{j}$, instead of directly controlling the joint angles.

To fully define controller (35), the feedback gain $k_{c}$ should be designed to achieve the desired motion. Considering the complex, nonlinear and under-actuated mathematical model of the robot, this task can be very challenging. However, one can use the model presented in (31) and run the simulation on uneven terrains and search for the desired value of $k_{c}$. To achieve this goal, we used the model in (31) and simulated the motion based on gait pattern (34) with $A_{p}=\frac{\pi}{4} \mathrm{rad}, \omega=\pi \mathrm{rad} / \mathrm{sec}$, $\Pi^{n}=\left[\frac{-2 \pi}{5}, \frac{-2 \pi}{5}, \ldots, \frac{-2 \pi}{5}\right]^{\mathrm{T}}$ and the controller shown in Fig. 8 in an environment with a stair type obstacle $(6 \mathrm{~cm}$ high, $11 \mathrm{~cm}$ wide) located $5 \mathrm{~cm}$ away from the head module. We increased the feedback gain $k_{c}$, incrementally by 0.1 from 0 to 15 and calculated 
the average velocity of the robot after $60 \mathrm{sec}$. The average velocity of the snake robot with each value of the feedback gain $k_{c}$ is shown in Fig. 9.

As it can be seen in Fig. 9, using (35) with $k_{c}=10.1$, the maximum forward speed is achieved, which is close to the average velocity obtained on the smooth surface. Moreover, the results showed that with $k_{c}=10.1$, the average forward speed of the robot is higher by 24.8 percent compared to $k_{c}=0$. This is an important result because as it can be seen from (35), with $k_{c}=0, \Pi$ converges to $\Pi^{n}$ independent of $\boldsymbol{Q}^{e}$, similar to the case when the open loop gait pattern (34) is being used. Hence, Fig. 9 shows that such closed-loop control strategy with $k_{c}=10.1$ results in a faster motion compared to gait pattern (34) with the same parameters but without feedback. Fig. 10 shows the snake robot climbing over the stair located on its path without prior knowledge about its location with control (35) with $M_{d}=$ $1 \frac{\mathrm{Nm}(\mathrm{sec})^{2}}{\mathrm{rad}}, D_{d}=10 \frac{\mathrm{Nm}(\mathrm{sec})}{\mathrm{rad}}, K_{d}=17.5 \frac{\mathrm{Nm}}{\mathrm{rad}}$ and both $k_{\boldsymbol{c}}=10.1$ and $k_{c}=0$.

It should be noted that to model the stair type obstacle, same spring-damper contact model as the previous section has been used. However, in addition to model the contact between the robot and the top side of the stair, contact forces exerted on the robot due to the collision to the side of the stair has also been included into the model. The reason is that there are cases that the robot cannot climb over the stair due to small amplitude of the wave, i.e. $A_{p}$, hence it might collide with the obstacle and if neglected can affect the results considerably. Moreover, as it is shown in Fig. 10 , the model has successfully been used to simulate the robot in contact with the environment at three different points, which was not possible using the contact modelling framework proposed in [19]. 
To obtain the simulation results in Fig. 10, $2 N+1$ points (the centre of mass of the links, robot joints, tip of the head and the tail) on the robot body have been checked at each step-time to determine the contact points and calculate the external forces. Unlike on smooth surfaces, in environments with sharp edge obstacles, there is a high possibility that the links touch the obstacle at the points other than these $2 N+1$ points. Such problem can be addressed by considering more candidate contact points to be checked at each step-time, which increases the computation burden of the simulation model. However, because this problem happens mostly due to the sharp edges of the obstacle touching the links, checking the distance between the candidate contact points and the sharp points of the obstacle (vertices of the squareshape obstacle) or considering a bounding a box around the obstacle and checking if the candidate contact points have touched the borders of the bounding box are other possible solutions. (See [40] for more details about the collision detection problem in simulation environments.)

To investigate the effectiveness of the proposed controller on the physical robot, the first experiment was conducted on the real robot based on (35) by setting $k_{c}=0$. However, the robot could not climb over the stair due to rolling over to one side. This issue could not be modeled by the presented modelling framework because the model only describes the motion in $\mathrm{XZ}$ plane and lateral forces in $\mathrm{Y}$ direction cannot be taken into account. This means that the value of the measured motor torque and the simulation results are valid as long as the side stability of the robot is guaranteed. This is not a major issue in pedal wave motion on smooth surfaces, because as the robot moves in a straight line, the projection of the centre of mass of the robot lies inside the convex hull of the contact points (all located on a straight line). Hence, the robot 
will not roll over to one side. However, when the robot is climbing over an obstacle, it could be in contact with four different surfaces, i.e., the ground, sides of the obstacle and top of the obstacle at the same time (see, [41] which gives more information about the similar issue in stable walking on uneven terrains). Hence, (see the video: https://www.youtube.com/watch?v=Up00yj3S2mQ) the snake robot can roll over to one side.

Finally, to evaluate the performance of the physical robot using the optimized controller, the designed controller based on (35) with $k_{c}=10.1$ was implemented on the physical snake robot, and the recorded motion is shown in Fig. 11. As can be seen in Fig. 11, the robot could successfully climb over the stair with the same dimensions as of the stair in the simulation, with the average velocity of $1.3 \mathrm{~cm} / \mathrm{sec}$. Moreover, as it can be seen in the experiment, unlike the case with $k_{c}=0$, the robot did not roll over to one side and successfully climbed over the obstacle due to the feedback signal $\boldsymbol{Q}^{e}$, which modulates the phase shift $\Pi$. Hence, unlike the case with $k_{\boldsymbol{c}}=0$, $\Pi$ deviated from $\Pi^{\mathbf{n}}$ based on the feedback signal, producing an adaptive pedal wave motion.

To improve the performance of the snake robot with the use of the presented controller, one way is to use the obtained value of $k_{c}$ from the simulation as the initial search point for experimentation based optimization methods, such as Bayesian optimization [42]. One can also consider pedal wave motion with an added lateral motion by installing additional servo motors and extend model (31) to further investigate the side stability of the robot during locomotion on uneven terrains. Another method, which could improve the performance of the controller, is to include 
other sensors, such as IMUs in addition to the torque sensors to achieve more adaptive and stable pedal wave motion.

\section{CONCLUSION}

In this paper introduced a general modelling framework for modelling modular snake robots with SEAs performing undulation in the vertical plane (pedal wave motion). Kinematics of such snake robot in vertical plane was obtained in a matrix form and Euler-Lagrange equations of motion were presented. Using the obtained model and with the help of a spring-damper contact model, pedal wave motion of the robot on smooth surfaces was simulated and the effect of changing friction was investigated, which supported by the experimentation results, showed that the forward velocity of the pedal wave motion of the snake robot is higher on the surfaces with high friction. In addition, to validate the model, the torque signal measured by the elastic elements embedded at each joint of the physical robot was compared with the simulation results, which showed that they bear a considerable resemblance, with expected discrepancies due to the simplified friction and contact models. Comparing the theoretical predictions with simulation-based physics engine can be considered as another method to verify the results in future works. Moreover, it is shown that this model is powerful enough to simulate snake locomotion on uneven terrains, simulating the robot motion climbing over a stair with the height of $6 \mathrm{~cm}$ and width of $11 \mathrm{~cm}$. Finally, an adaptive gait based controller using the measured torque feedback of the elastic element was proposed and optimized using a search-based method. The controller was implemented on the physical robot, which showed the robot can 
successfully climb over the obstacle without any prior knowledge about the exact location of the obstacle.

\section{APPENDIX}

The matrices of dynamical model (31) can be obtained as follows:

$$
\begin{aligned}
M^{L}(\boldsymbol{q})=\left(\mathcal{B}_{1}^{l}\right. & \left.+\mathcal{A}^{l} \mathcal{S}_{\theta} \mathcal{C}\right)^{T} \mathcal{M}^{l}\left(\mathcal{B}_{1}^{l}+\mathcal{A}^{l} \mathcal{S}_{\theta} \mathcal{C}\right) \\
& +\left(\mathcal{B}_{2}^{l}-\mathcal{A}^{l} \mathcal{C}_{\theta} \mathcal{C}\right)^{T} \mathcal{M}^{l}\left(\mathcal{B}_{2}^{l}-\mathcal{A}^{l} \mathcal{C}_{\theta} \mathcal{C}\right)+\mathcal{C}^{T} \mathcal{J}^{l} \mathcal{C} \\
M^{R}(\boldsymbol{q})=\left(\mathcal{B}_{1}^{r}\right. & \left.+\mathcal{A}^{r} \mathcal{S}_{\theta} \mathcal{C}\right)^{T} \mathcal{M}^{r}\left(\mathcal{B}_{1}^{r}+\mathcal{A}^{r} \mathcal{S}_{\theta} \mathcal{C}\right) \\
& +\left(\mathcal{B}_{2}^{r}-\mathcal{A}^{r} \mathcal{C}_{\theta} \mathcal{C}\right)^{T} \mathcal{M}^{r}\left(\mathcal{B}_{2}^{r}-\mathcal{A}^{r} \mathcal{C}_{\theta} \mathcal{C}\right)
\end{aligned}
$$

The matrix of centripetal forces can be found as below:

$$
\begin{aligned}
& \boldsymbol{C}^{L}(\boldsymbol{q}, \dot{\boldsymbol{q}})=\boldsymbol{C C}\left(\mathcal{B}_{1}^{l}, \mathcal{B}_{2}^{l}, \mathcal{A}^{l}, \mathcal{M}^{l}, \boldsymbol{q}_{\mathbf{1}}, \dot{\boldsymbol{q}}_{1}\right) \\
& \boldsymbol{C}^{R}(\boldsymbol{q}, \dot{\boldsymbol{q}})=\boldsymbol{C C}\left(\mathcal{B}_{1}^{r}, \mathcal{B}_{2}^{r}, \mathcal{A}^{r}, \mathcal{M}^{r}, \boldsymbol{q}_{1}, \dot{q}_{\mathbf{1}}\right)
\end{aligned}
$$

where

$$
\begin{aligned}
& \boldsymbol{C C}\left(\mathcal{B}_{1}^{l}, \mathcal{B}_{2}^{l}, \mathcal{A}^{l}, \boldsymbol{q}_{1}, \dot{\boldsymbol{q}}_{\mathbf{1}}\right)= \\
& +\left\{\mathcal{B}_{1}^{l^{T}} \mathcal{M}^{l} \mathcal{A}^{l} \mathcal{C}_{\theta} \operatorname{diag}\left(\dot{\boldsymbol{\theta}}^{l}\right) \mathcal{C}+\mathcal{C}^{T} \mathcal{C}_{\theta} \operatorname{diag}\left(\dot{\boldsymbol{\theta}}^{l}\right) \mathcal{A}^{l^{T}} \mathcal{M}^{l} \mathcal{B}_{1}^{l}\right. \\
& +\mathcal{C}^{T} \mathcal{S}_{\theta} \mathcal{A}^{l^{T}} \mathcal{M}^{l} \mathcal{A}^{l} \mathcal{C}_{\theta} \operatorname{diag}\left(\dot{\boldsymbol{\theta}}^{l}\right) \mathcal{C} \\
& +\mathcal{C}^{T} \mathcal{C}_{\theta} \operatorname{diag}\left(\dot{\boldsymbol{\theta}}^{l}\right) \mathcal{A}^{l^{T}} \mathcal{M}^{l} \mathcal{A}^{l} \mathcal{S}_{\theta} \mathcal{C} \\
& +\mathcal{B}_{2}^{l^{T}} \mathcal{M}^{l} \mathcal{A}^{l} \delta_{\theta} \operatorname{diag}\left(\dot{\boldsymbol{\theta}}^{l}\right) \mathcal{C}+\mathcal{C}^{T} \mathcal{S}_{\theta} \operatorname{diag}\left(\dot{\boldsymbol{\theta}}^{l}\right) \mathcal{A}^{l^{T}} \mathcal{M}^{l} \mathcal{B}_{2}^{l} \\
& -\mathcal{C}^{T} \mathcal{C}_{\theta} \mathcal{A}^{l^{T}} \mathcal{M}^{l} \mathcal{A}^{l} \mathcal{S}_{\theta} \operatorname{diag}\left(\dot{\boldsymbol{\theta}}^{l}\right) \mathcal{C} \\
& \left.-\mathcal{C}^{T} \mathcal{S}_{\theta} \operatorname{diag}\left(\dot{\boldsymbol{\theta}}^{l}\right) \mathcal{A}^{l^{T}} \mathcal{M}^{l} \mathcal{A}^{l} \mathcal{C}_{\theta} \mathcal{C}\right\} \dot{\boldsymbol{q}}_{\mathbf{1}} \\
& +\left[\begin{array}{c}
\dot{q}_{1}^{T} B_{1}{ }^{T} \mathcal{M}^{l} \mathcal{A}^{l} \mathcal{C}_{\theta} \operatorname{diag}\left(C_{1}\right) \dot{\theta}^{l} \\
\dot{q}_{1}^{T} B_{1}{ }^{T} \mathcal{M}^{l} \mathcal{A}^{l} \mathcal{C}_{\theta} \operatorname{diag}\left(C_{2}\right) \dot{\theta}^{l} \\
\vdots \\
\dot{q}_{1}^{T} B_{1}{ }^{T} \mathcal{M}^{l} \mathcal{A}^{l} \mathcal{C}_{\theta} \operatorname{diag}\left(C_{2 N+1}\right) \dot{\theta}^{l}
\end{array}\right]+\left[\begin{array}{c}
\dot{\theta}^{l^{T}} \mathcal{C}_{\theta} \operatorname{diag}\left(C_{1}\right) \mathcal{A}^{l^{T}} \mathcal{M}^{l} \mathcal{B}_{1}^{l} \dot{q}_{1} \\
\dot{\theta}^{l^{T}} \mathcal{C}_{\theta} \operatorname{diag}\left(C_{2}\right) \mathcal{A}^{l^{T}} \mathcal{M}^{l} \mathcal{B}_{1}^{l} \dot{q}_{1} \\
\vdots \\
\dot{\theta}^{{ }^{T}} \mathcal{C}_{\theta} \operatorname{diag}\left(C_{2 N+1}\right) \mathcal{A}^{l^{T}} \mathcal{M}^{l} \mathcal{B}_{1}^{l} \dot{q}_{1}
\end{array}\right]
\end{aligned}
$$


$+\left[\begin{array}{c}\dot{\theta}^{l^{T}}\left(\mathcal{C}_{\theta} \operatorname{diag}\left(C_{1}\right) \mathcal{A}^{l^{T}} \mathcal{M}^{l} \mathcal{A}^{l} \delta_{\theta}+\delta_{\theta} \mathcal{A}^{l^{T}} \mathcal{M}^{l} \mathcal{A}^{l} \mathcal{C}_{\theta} \operatorname{diag}\left(C_{1}\right)\right) \dot{\theta}^{l} \\ \dot{\theta}^{l^{T}}\left(\mathcal{C}_{\theta} \operatorname{diag}\left(C_{2}\right) \mathcal{A}^{l^{T}} \mathcal{M}^{l} \mathcal{A}^{l} \delta_{\theta}+\delta_{\theta} \mathcal{A}^{l^{T}} \mathcal{M}^{l} \mathcal{A}^{l} \mathcal{C}_{\theta} \operatorname{diag}\left(C_{2}\right)\right) \dot{\theta}^{l} \\ \vdots \\ \dot{\theta}^{l^{T}}\left(\mathcal{C}_{\theta} \operatorname{diag}\left(C_{2 N+1}\right) \mathcal{A}^{l^{T}} \mathcal{M}^{l} \mathcal{A}^{l} \delta_{\theta}+\delta_{\theta} \mathcal{A}^{l^{T}} \mathcal{M}^{l} \mathcal{A}^{l} \mathcal{C}_{\theta} \operatorname{diag}\left(C_{2 N+1}\right)\right) \dot{\theta}^{l}\end{array}\right]$ $+\left[\begin{array}{c}\dot{\boldsymbol{q}}_{1}^{T} \mathcal{B}_{2}^{l_{2}^{T}} \mathcal{M}^{l} \mathcal{A}^{l} \boldsymbol{S}_{\theta} \operatorname{diag}\left(C_{1}\right) \dot{\theta}^{l} \\ \dot{\boldsymbol{q}}_{1}^{T} \mathcal{B}_{2}^{l^{T}} \mathcal{M}^{l} \mathcal{A}^{l} \boldsymbol{S}_{\theta} \operatorname{diag}\left(C_{2}\right) \dot{\theta}^{l} \\ \vdots \\ \dot{\boldsymbol{q}}_{1}^{T} \mathcal{B}_{2}^{l^{T}} \mathcal{M}^{l} \mathcal{A}^{l} \delta_{\theta} \operatorname{diag}\left(C_{2 N+1}\right) \dot{\theta}^{l}\end{array}\right]+\left[\begin{array}{c}\dot{\theta}^{l^{T}} \delta_{\theta} \operatorname{diag}\left(C_{1}\right) \mathcal{A}^{l^{T}} \mathcal{M}^{l} \mathcal{B}_{2}^{l} \dot{\boldsymbol{q}}_{1} \\ \dot{\theta}^{l^{T}} \delta_{\theta} \operatorname{diag}\left(C_{2}\right) \mathcal{A}^{l^{T}} \mathcal{M}^{l} \mathcal{B}_{2}^{l} \dot{\boldsymbol{q}}_{1} \\ \vdots \\ \dot{\theta}^{l^{T}} \delta_{\theta} \operatorname{diag}\left(C_{2 N+1}\right) \mathcal{A}^{l^{T}} \mathcal{M}^{l} \mathcal{B}_{2}^{l} \dot{\boldsymbol{q}}_{1}\end{array}\right]$ $-\left[\begin{array}{c}\dot{\theta}^{l^{T}}\left(\delta_{\theta} \operatorname{diag}\left(C_{1}\right) \mathcal{A}^{l^{T}} \mathcal{M}^{l} \mathcal{A}^{l} \mathcal{C}_{\theta}+\mathcal{C}_{\theta} \mathcal{A}^{l^{T}} \mathcal{M}^{l} \mathcal{A}^{l} \delta_{\theta} \operatorname{diag}\left(C_{1}\right)\right) \dot{\theta}^{l} \\ \dot{\theta}^{l^{T}}\left(\delta_{\theta} \operatorname{diag}\left(C_{2}\right) \mathcal{A}^{l^{T}} \mathcal{M}^{l} \mathcal{A}^{l} \mathcal{C}_{\theta}+\mathcal{C}_{\theta} \mathcal{A}^{l^{T}} \mathcal{M}^{l} \mathcal{A}^{l} s_{\theta} \operatorname{diag}\left(C_{2}\right)\right) \dot{\theta}^{l} \\ \vdots \\ \dot{\theta}^{l^{T}}\left(\delta_{\theta} \operatorname{diag}\left(C_{2 N+1}\right) \mathcal{A}^{l^{T}} \mathcal{M}^{l} \mathcal{A}^{l} \mathcal{C}_{\theta}+\mathcal{C}_{\theta} \mathcal{A}^{l^{T}} \mathcal{M}^{l} \mathcal{A}^{l} s_{\theta} \operatorname{diag}\left(C_{2 N+1}\right)\right) \dot{\theta}^{l}\end{array}\right]$, $\left[C_{1}, C_{2}, \ldots, C_{2 N+1}\right]=\mathcal{C}_{N \times(2 N+1)}=\left[\begin{array}{lll}\mathcal{F} & V^{l} & 0_{N \times 2}\end{array}\right]$,

and $\dot{\boldsymbol{q}}_{\mathbf{1}}$ is the first $N+2$ elements of state vector $\dot{\boldsymbol{q}}$. 


\section{NOMENCLATURE}

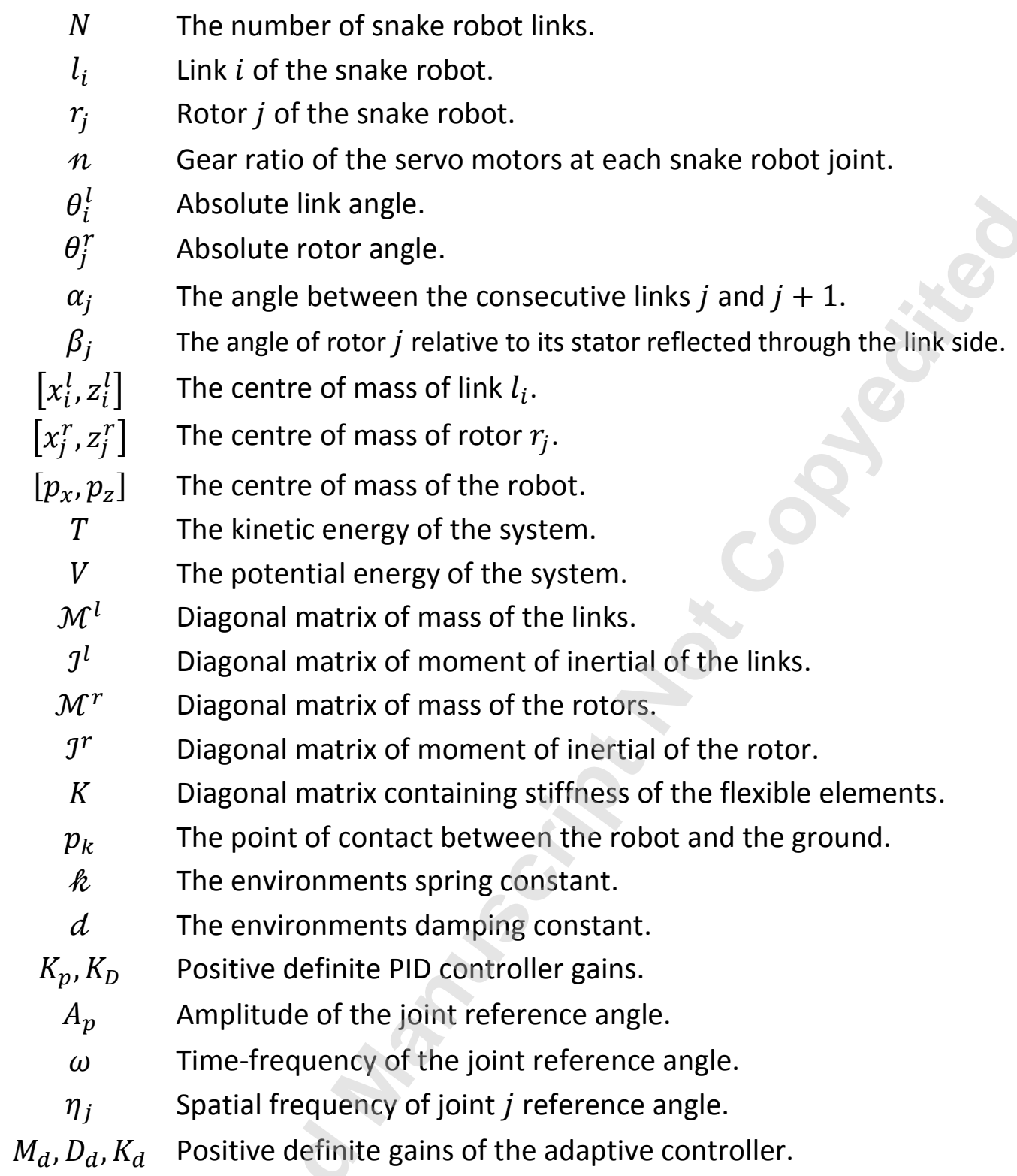




\section{REFERENCES}

[1] Hirose, S., 1993, "Biologically Inspired Robot," Oxford University Press, New York, NY, USA.

[2] Zarrouk, D., and Shoham, M., 2012, "Analysis and Design of One Degree of Freedom Worm Robots for Locomotion on Rigid and Compliant Terrain," J. Mech. Des., 134(2), p. 021010.

[3] Yamada, H., Takaoka, S., and Hirose, S., 2013, "A Snake-like Robot for RealWorld Inspection Applications (the Design and Control of a Practical Active Cord Mechanism)," Adv. Robot., 27(1), pp. 47-60.

[4] Liljebäck, P., Pettersen, K. Y., Stavdahl, O., and Gravdahl, J. T., 2011, "Experimental Investigation of Obstacle-Aided Locomotion with a Snake Robot," IEEE Trans. Robot., 27(4), pp. 792-800.

[5] Hu, D. L., Nirody, J., Scott, T., and Shelley, M. J., 2009, "The Mechanics of Slithering Locomotion," Proc. Natl. Acad. Sci., 106(25), pp. 10081-10085.

[6] Marvi, H., Hu, D. L. D. L., Home, E., Mosauer, W., Bogert, C., Lissman, H., Walton, M., Jayne, B., Bennett, A. A., Alexander, R., Keller, J., Falkovitz, M., Gray, S., Zimmermann, K., Zeidis, I., Mehrtens, J., Mosauer, W., Dormand, J., Prince, P., Schmidt-Nielsen, K., Gans, C., Gasc, J., Jayne, B., Davis, J., Renous, S., Hofling, E., Gasc, J., Berrigan, D., Pepin, D., Brackenbury, J., Brackenbury, J., Joos, B., Casey, T., Quillin, K., and Quillin, K., 2013, "Snakes Mimic Earthworms: Propulsion Using Rectilinear Travelling Waves.," J. R. Soc. Interface, 10(84), pp. 20130188.

[7] Yamada, H., and Hirose, S., 2010, "Steering of Pedal Wave of a Snake-like Robot by Superposition of Curvatures," 2010 IEEE/RSJ International Conference on Intelligent Robots and Systems, IEEE, pp. 419-424.

[8] Gart, S. W., Mitchel, T. W., and Li, C., 2019, "Snakes Partition Their Body to Traverse Large Steps Stably," J. Exp. Biol., p. jeb.185991.

[9] Hopkins, J. K. K., Spranklin, B. W. W., and Gupta, S. K. K., 2009, "A Survey of Snake-Inspired Robot Designs," Bioinspir Biomim, 4(2), p. 21001.

[10] Tanaka, M., and Matsuno, F., 2014, "Control of Snake Robots with Switching Constraints: Trajectory Tracking with Moving Obstacle," Adv. Robot., 28(6), pp. 415-429.

[11] Pettersen, K. Y., 2017, “Snake Robots," Annu. Rev. Control, 44, pp. 19-44.

[12] Filippov, A. E., and Gorb, S. N., 2016, "Modelling of the Frictional Behaviour of the Snake Skin Covered by Anisotropic Surface Nanostructures," Sci. Rep., 6(August 2015), p. 23539.

[13] Liljebäck, P., Pettersen, K. Y., Stavdahl, O., and Gravdahl, J. T., 2010, "Hybrid Modelling and Control of Obstacle-Aided Snake Robot Locomotion," IEEE Trans. Robot., 26(5), pp. 781-799.

[14] Transeth, A. A., Leine, R. I., Glocker, C., Pettersen, K. Y., and Liljebäck, P., 2008, "Snake Robot Obstacle-Aided Locomotion: Modeling, Simulations, and 
Experiments," IEEE Trans. Robot., 24(1), pp. 88-104.

[15] Kano, T., Sato, T., Kobayashi, R., and Ishiguro, A., 2012, "Local Reflexive Mechanisms Essential for Snakes' Scaffold-Based Locomotion," Bioinspir Biomim, 7(4), p. 46008.

[16] Rollinson, D., and Choset, H., 2016, "Pipe Network Locomotion with a Snake Robot," J. F. Robot., 33(3), pp. 322-336.

[17] Li, G., Li, W., Zhang, J., and Zhang, H., 2015, "Analysis and Design of Asymmetric Oscillation for Caterpillar-Like Locomotion," J. Bionic Eng., 12(2), pp. 190-203.

[18] Chen, L., Ma, S., Wang, Y., Li, B., and Duan, D., 2007, “Design and Modelling of a Snake Robot in Traveling Wave Locomotion," Mech. Mach. Theory, 42(12), pp. 1632-1642.

[19] Akbarzadeh, A., and Kalani, H., 2012, "Design and Modeling of a Snake Robot Based on Worm-Like Locomotion," Adv. Robot., 26(5-6), pp. 537-560.

[20] Sato, T., Kano, T., and Ishiguro, A., 2012, "A Decentralized Control Scheme for an Effective Coordination of Phasic and Tonic Control in a Snake-like Robot," Bioinspir. Biomim., 7(1), p. 016005.

[21] Koopaee, M. J., Pretty, C., Classens, K., and Chen, X., 2019, “Dynamical Modelling and Control of Snake-like Motion in Vertical Plane for Locomotion in Unstructured Environments," Accepted, proceedings of IDETC/CIE, Anaheim, California, USA, August 18-21, 2019, ASME Paper No. IDETC2019-97227.

[22] Koopaee, M. J, Bal, S., Pretty, C., and Chen, X., 2019, "Design and Development of a Wheel-less Snake Robot with Active Stiffness Control for Adaptive Pedal Wave Locomotion," J. Bionic Eng., 16(4), pp. 593-607.

[23] Chen, Y.-H., and Lan, C.-C., 2012, "An Adjustable Constant-Force Mechanism for Adaptive End-Effector Operations," J. Mech. Des., 134(3), p. 031005.

[24] Rollinson, D., Bilgen, Y., Brown, B., Enner, F., Ford, S., Layton, C., Rembisz, J., Schwerin, M., Willig, A., Velagapudi, P., and Choset, H., 2014, "Design and Architecture of a Series Elastic Snake Robot," IEEE International Conference on Intelligent Robots and Systems.

[25] Liljebäck, P., Pettersen, K. Y., Stavdahl, O., and Gravdahl, J. T., 2012, Snake Robots: Modelling, Mechatronics, and Control, Springer-Verlag, London, UK.

[26] Liljebäck, P., Stavdahl, Ø., Pettersen, K. Y., and Gravdahl, J. T., 2012, “A Modular and Waterproof Snake Robot Joint Mechanism with a Novel Force/Torque Sensor," IEEE Int. Conf. Intell. Robot. Syst., pp. 4898-4905.

[27] Takaoka, S., Yamada, H., and Hirose, S., 2011, "Snake-like Active Wheel Robot ACM-R4.1 with Joint Torque Sensor and Limiter," IEEE Int. Conf. Intell. Robot. Syst., pp. 1081-1086.

[28] Pratt, G. A., and Williamson, M. M., "Series Elastic Actuators," Proceedings 1995 IEEE/RSJ International Conference on Intelligent Robots and Systems. Human Robot Interaction and Cooperative Robots, IEEE Comput. Soc. Press, pp. 399406. 
[29] Travers, M., Whitman, J., and Choset, H., 2018, "Shape-Based Coordination in Locomotion Control," Int. J. Rob. Res., pp. 1253-1268.

[30] Marvi, H., Bridges, J., and Hu, D. L., 2013, "Snakes Mimic Earthworms: Propulsion Using Rectilinear Travelling Waves," J. R. Soc. Interface, 10(84).

[31] Bogert, C. M., 1947, "Rectilinear Locomotion in Snakes," Copeia, 1947(4), pp. 253-254.

[32] Mosauer, W., 1930, "A Note on the Sidewinding Locomotion of Snakes," Am. Nat., 64(691), pp. 179-183.

[33] Kano, T., Watanabe, Y., Satake, F., and Ishiguro, A., 2014, "DecentralizedControlled Multi-Terrain Robot Inspired by Flatworm Locomotion," Adv. Robot., 28(7), pp. 523-531.

[34] Mosauer, W., 1932, "On the Locomotion of Snakes," Science, 76(1982), pp. 583-585.

[35] Mohammadi, A., Rezapour, E., Maggiore, M., and Pettersen, K. Y. K. Y., 2016, "Maneuvering Control of Planar Snake Robots Using Virtual Holonomic Constraints," IEEE Trans. Control Syst. Technol., 24(3), pp. 884-899.

[36] Kandhari, A., Huang, Y., Daltorio, K. A., Chiel, H. J., and Quinn, R. D., 2018, “Body Stiffness in Orthogonal Directions Oppositely Affects Worm-like Robot Turning and Straight-Line Locomotion," Bioinspir. Biomim., 13(2), p. 026003.

[37] De Luca, A., and Book, W., 2008, "Robots with Flexible Elements," Springer Handbook of Robotics, B. Siciliano, O. Khatib, Springer-Verlag, Heidelberg, Germany, pp. 287-319.

[38] Nicosia, S., and Tomei, P., 1991, "A PD Control Law for Trajectory Tracking of Flexible Joint Robots," IFAC Proc, 24(9), pp. 357-362.

[39] Calanca, A., Muradore, R., and Fiorini, P., 2017, "Impedance Control of Series Elastic Actuators: Passivity and Acceleration-Based Control," Mechatronics, 47, pp. 37-48.

[40] Kim, Y. J., Lin, M. C., and Manocha, D., 2017, "Collision Detection," Humanoid Robotics: A Reference, Springer Netherlands, Dordrecht, pp. 1-24.

[41] Sardain, P., and Bessonnet, G., 2004, "Forces Acting on a Biped Robot. Center of Pressure-Zero Moment Point," IEEE Trans. Syst. Man, Cybern. - Part A Syst. Humans, 34(5), pp. 630-637.

[42] Tesch, M., Schneider, J., and Choset, H., 2011, "Using Response Surfaces and Expected Improvement to Optimize Snake Robot Gait Parameters," 2011 IEEE/RSJ International Conference on Intelligent Robots and Systems, IEEE, pp. 1069-1074. 


\section{LIST OF FIGURES}

Fig. 1 Snake robot progression with pedal wave motion with $A_{p}=\frac{\pi}{4} \mathbf{r a d}, \omega=\pi \mathrm{rad} / \mathrm{sec}$ and $\boldsymbol{\eta}=\frac{-2.2 \pi}{5} \boldsymbol{r a d}$ first on a high friction and then on a low friction surface.

Fig. 2 The components of each robot module. (a) is the servomotor (DSR-0101), (b) is the magnet, (c) is the elastic element, (d) is the magnetic encoder and (e) is the sensor holder.

Fig. 3 The snake robot body shape in vertical plane XZ.

Fig. 4 The defined parameters of $\boldsymbol{j}^{\text {th }}$ robot module.

Fig. 5 The force diagram showing the contact forces exerted to the robot module.

Fig. 6 Simulated pedal wave motion with $A_{p}=\frac{\pi}{4} \mathrm{rad}, \omega=\pi \mathrm{rad} / \mathrm{sec}$ and $\boldsymbol{\eta}=\frac{-2.2 \pi}{5} \mathrm{rad}$ with the average velocity of $2.18 \mathrm{~cm} / \mathrm{s}$.

Fig. 7 Comparison between the torque signals experimentally measured by the elastic element of the first joint and the same quantity obtained from the simulation.

Fig. 8 Block diagram of the gait based adaptive controller.

Fig. 9 Effect of $\boldsymbol{k}_{\boldsymbol{c}}$ on forward speed of the robot while climbing over the stair with height $6 \mathrm{~cm}$ and width $11 \mathrm{~cm}$.

Fig. 10 Pedal wave motion of the snake robot with flexible joints with $\boldsymbol{k}_{\boldsymbol{c}}=\mathbf{0}$ (red) and $\boldsymbol{k}_{\boldsymbol{c}}=$ 10.1 (blue) while climbing over a stair type obstacle with the height of $6 \mathrm{~cm}$ and width of $11 \mathrm{~cm}$ with the average velocity of $2.13 \mathrm{~cm} / \mathrm{sec}$.

Fig. 11 The Physical snake robot climbing over the obstacle with height $6 \mathrm{~cm}$ and width $11 \mathrm{~cm}$, with average velocity of $1.3 \mathrm{~cm} / \mathrm{sec}$. 

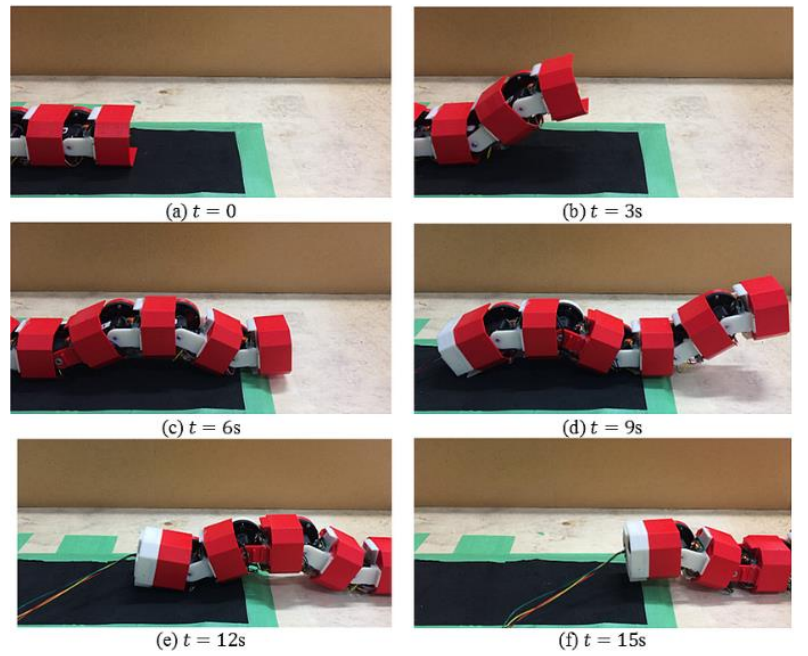

Fig. 1 Snake robot progression with pedal wave motion with $A_{p}=\frac{\pi}{4} \mathrm{rad}, \omega=\pi \mathrm{rad} / \mathrm{sec}$ and $\eta=\frac{-2.2 \pi}{5} \mathrm{rad}$ first on a high friction and then on a low friction surface. 


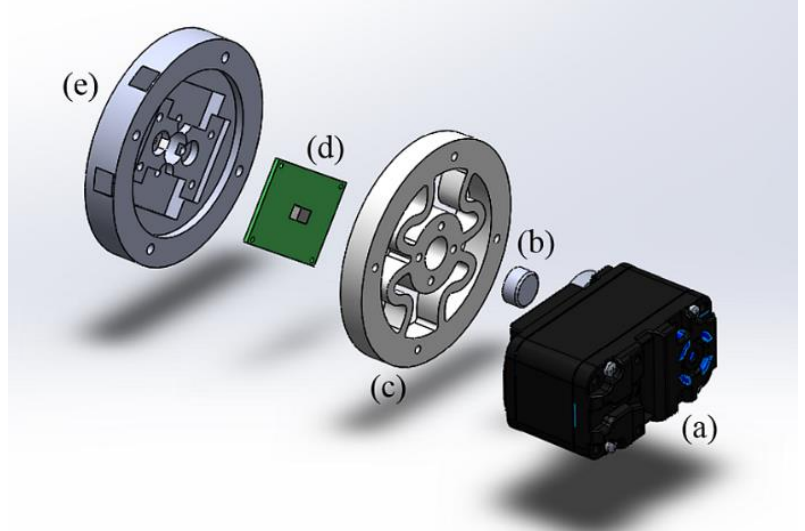

Fig. 2 The components of each robot module. (a) is the servomotor (DSR-0101), (b) is the magnet, (c) is the elastic element, (d) is the magnetic encoder and (e) is the sensor holder. 

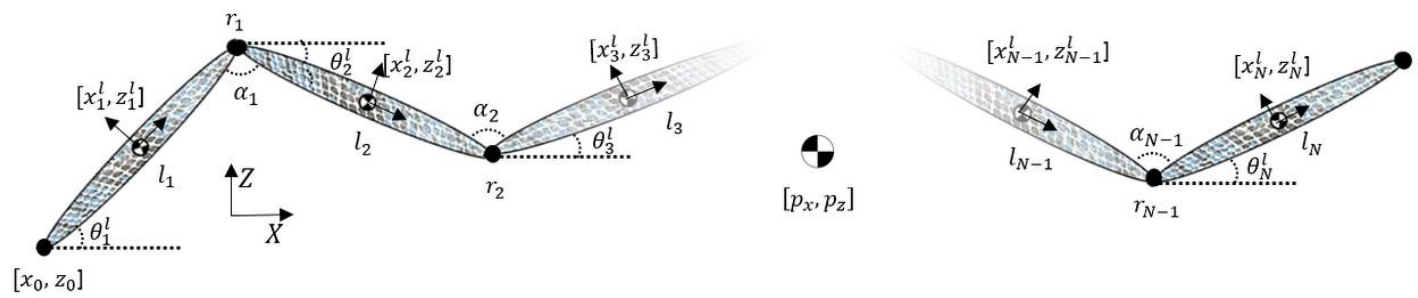

Fig. 3 The snake robot body shape in vertical plane XZ. 


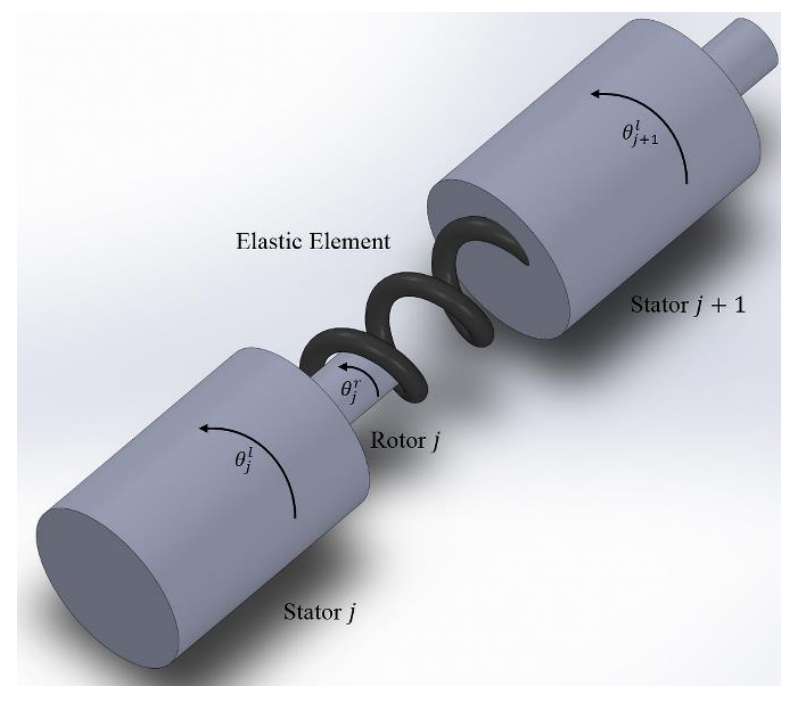

Fig. 4 The defined parameters of $j^{\text {th }}$ robot module. 


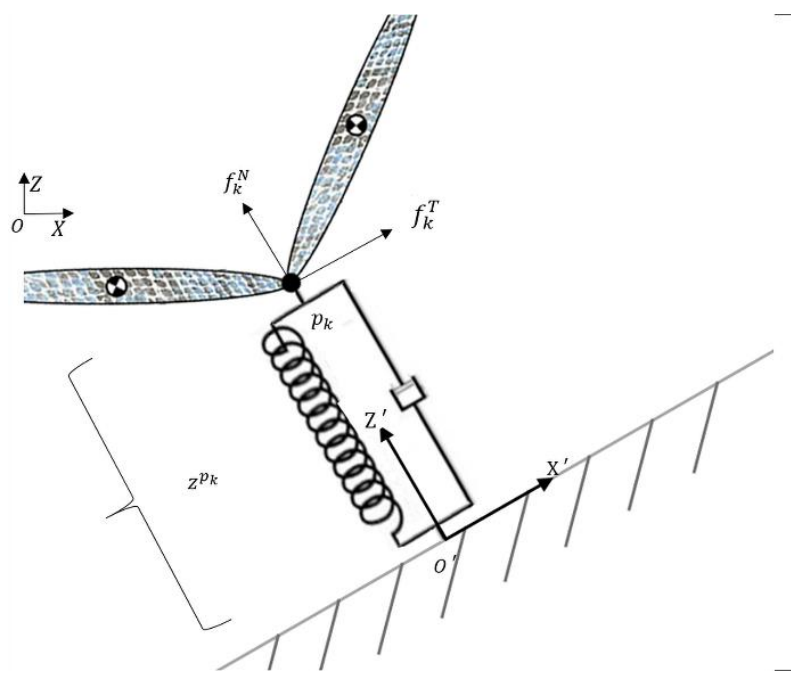

Fig. 5 The force diagram showing the contact forces exerted to the robot module. 


\begin{tabular}{l|lll|l|}
$-0.5 m$ & 0 & & $0.5 m$ & $1 \mathrm{~m}$ \\
\hline
\end{tabular}

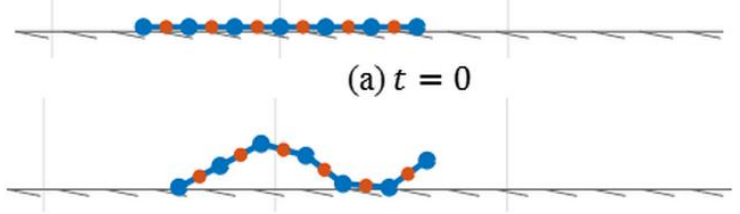

(b) $t=3 \mathrm{~s}$

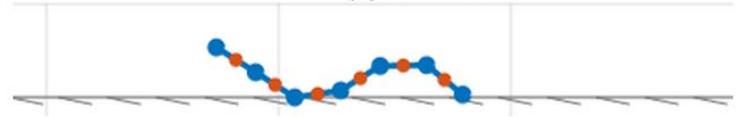

(c) $t=6 \mathrm{~s}$

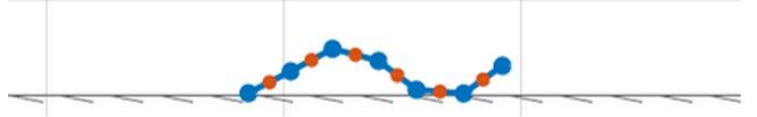

(d) $t=9 \mathrm{~s}$

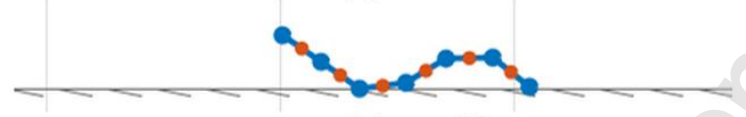

(e) $t=12 \mathrm{~s}$

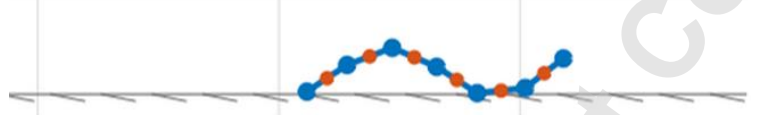

(f) $t=15$

Fig. 6 Simulated pedal wave motion with $A_{p}=\frac{\pi}{4} \mathrm{rad}, \omega=\pi \mathrm{rad} / \mathrm{sec}$ and $\eta=$ $\frac{-2.2 \pi}{5} \mathrm{rad}$ with the average velocity of $2.18 \mathrm{~cm} / \mathrm{s}$. 


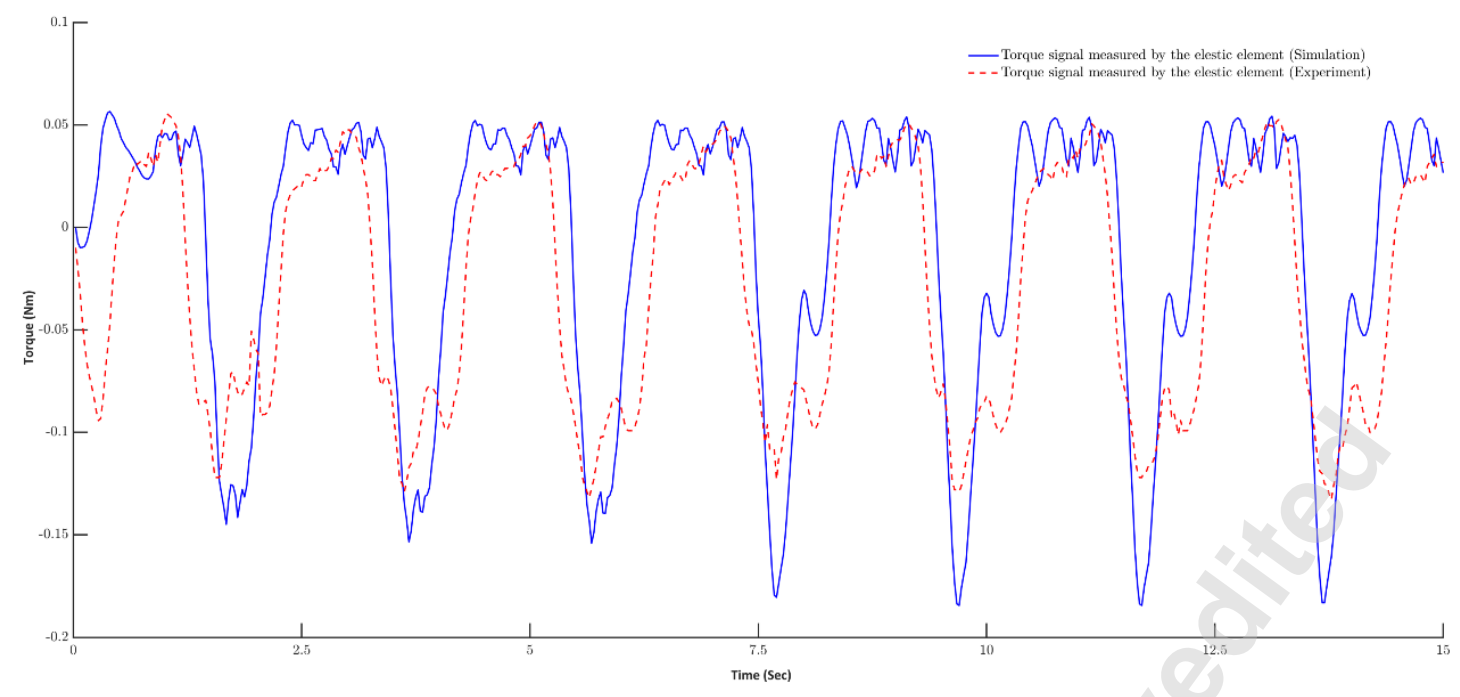

Fig. 7 Comparison between the torque signals experimentally measured by the elastic element of the first joint and the same quantity obtained from the simulation. 


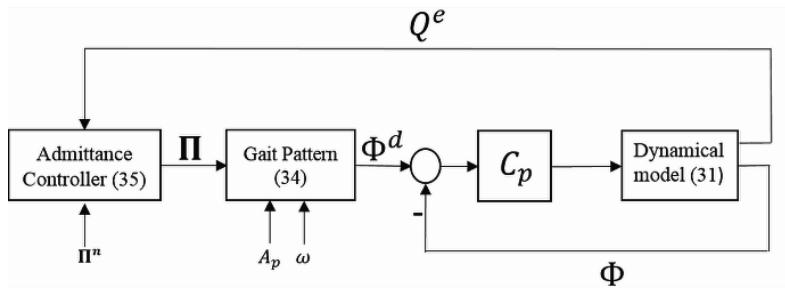

Fig. 8 Block diagram of the gait based adaptive controller. 


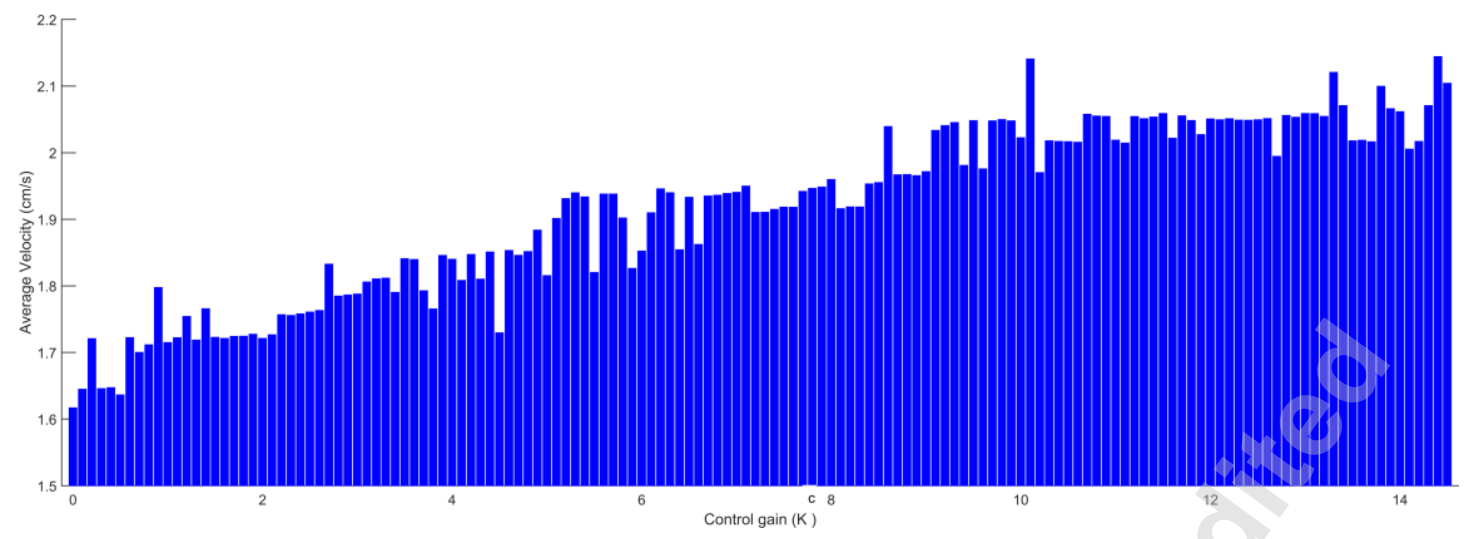

Fig. 9 Effect of $\boldsymbol{k}_{c}$ on forward speed of the robot while climbing over the stair with height $6 \mathrm{~cm}$ and width $11 \mathrm{~cm}$. 


\begin{tabular}{|c|c|c|c|}
\hline $0.5 \mathrm{~m}$ & 0 & $0.5 \mathrm{~m}$ & $1 \mathrm{~m}$ \\
\hline
\end{tabular}

(a) $\mathrm{t}=0$

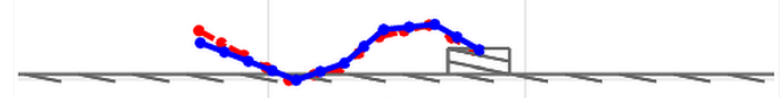

(b) $\mathrm{t}=6 \mathrm{~s}$

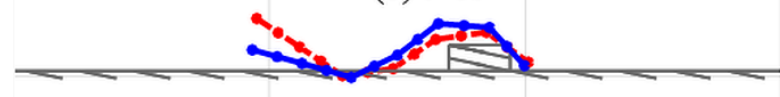

(c) $\mathrm{t}=12 \mathrm{~s}$

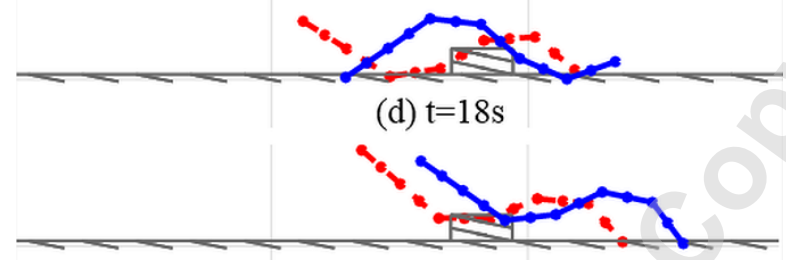

(e) $t=24 \mathrm{~s}$

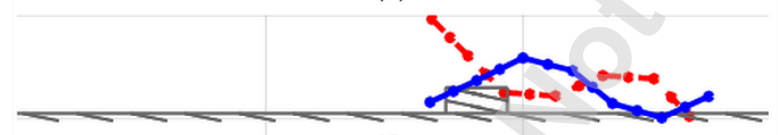

(f) $\mathrm{t}=30 \mathrm{~s}$

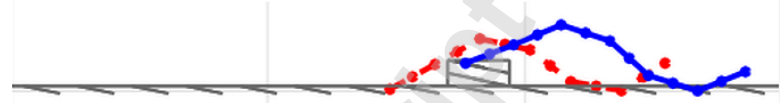

(f) $t=36 \mathrm{~s}$

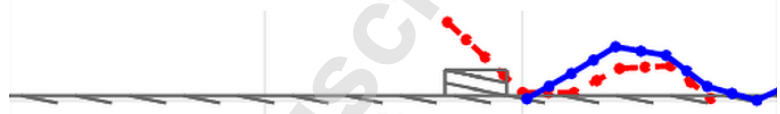

(h) $\mathrm{t}=42 \mathrm{~s}$

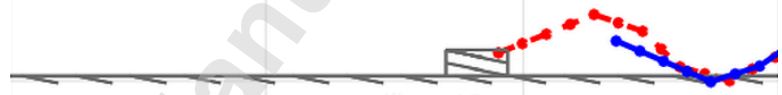

(i) $\mathrm{t}=48 \mathrm{~s}$

Fig. 10 Pedal wave motion of the snake robot with flexible joints with $\boldsymbol{k}_{c}=\mathbf{0}$ (dashed red line) and $\boldsymbol{k}_{c}=10.1$ (solid blue line) while climbing over a stair type obstacle with the height of $6 \mathrm{~cm}$ and width of $11 \mathrm{~cm}$ with the average velocity of $2.13 \mathrm{~cm} / \mathrm{sec}$. 

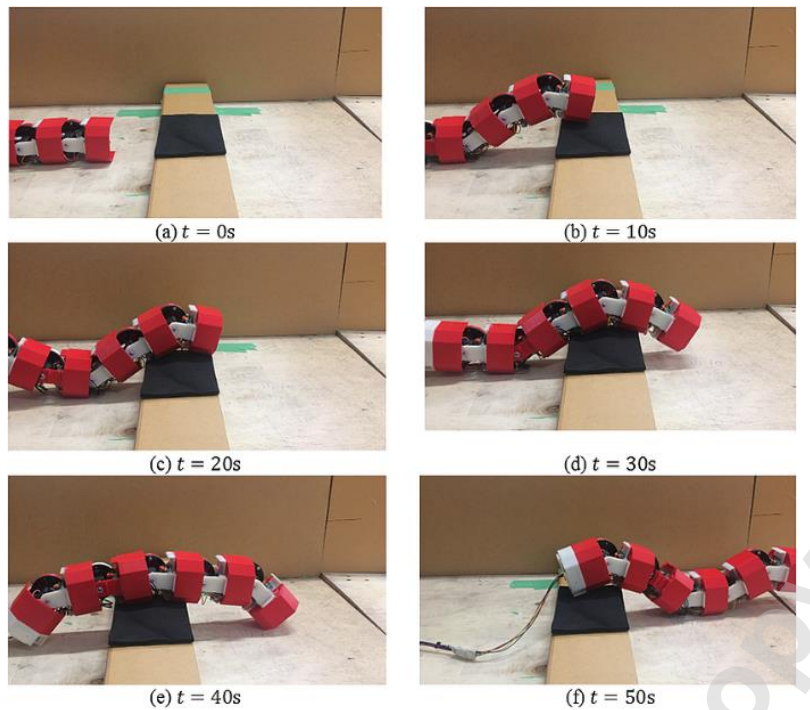

Fig. 11 The Physical snake robot climbing over the obstacle with height $6 \mathrm{~cm}$ and width $11 \mathrm{~cm}$, with average velocity of $1.3 \mathrm{~cm} / \mathrm{sec}$. 